Review Article

\title{
Prevalence of Hypothyroidism in Pregnant Women in India: A Meta-Analysis of Observational Studies
}

\author{
Vikas Yadav $\mathbb{D D}^{1}{ }^{1}$ Deepti Dabar $\mathbb{D}^{2},{ }^{2}$ Akhil D. Goel $\mathbb{D}^{\circ},{ }^{3}$ Mohan Bairwa $\left(\mathbb{D},{ }^{4}\right.$ Akanksha Sood $\left(\mathbb{D},{ }^{5}\right.$ \\ Pankaj Prasad (D, ${ }^{2}$ Sanjay S. Agarwal, ${ }^{1}$ and Sunil Nandeshwar ${ }^{1}$
}

${ }^{1}$ Atal Bihari Vajpayee Government Medical College, Vidisha, India

${ }^{2}$ All India Institute of Medical Sciences, Bhopal, India

${ }^{3}$ All India Institute of Medical Sciences, Jodhpur, India

${ }^{4}$ All India Institute of Medical Sciences, New Delhi, India

${ }^{5}$ St. Mary's Hospital, Oxford Road, Manchester, UK

Correspondence should be addressed to Deepti Dabar; deepti.dabar@gmail.com

Received 7 January 2021; Revised 1 February 2021; Accepted 3 February 2021; Published 19 February 2021

Academic Editor: Brendan C. Stack Jr.

Copyright ( $\odot 2021$ Vikas Yadav et al. This is an open access article distributed under the Creative Commons Attribution License, which permits unrestricted use, distribution, and reproduction in any medium, provided the original work is properly cited.

\begin{abstract}
Introduction. This meta-analysis was conducted to estimate the prevalence of hypothyroidism among pregnant women in India. Methods. We searched PubMed, Web of Science, Scopus, Google Scholar, and Shodhganga (Indian thesis repository) for observational studies, providing prevalence of hypothyroidism among pregnant women in India. Systematic study selection and data extraction procedures were followed. Quality assessment of each study was done using JBI critical appraisal checklist. The random effects model was used for pooling the effect sizes. Publication bias was assessed using the funnel plot and rank correlation test. $I^{2}$ statistics was used to measure heterogeneity across the studies. Heterogeneity in the pooled estimates was further explored with subgroup analyses and meta-regression analysis. Results. Sixty-one studies were found eligible and included in this review. The pooled estimate of the prevalence of hypothyroidism in pregnant women was $11.07 \%$ (95\% CI: 8.79-13.84, $\left.I^{2}=99 \%\right)$. Pooled prevalence estimates of subclinical and overt hypothyroidism are 9.51\% (95\% CI: $\left.7.48-12.04, I^{2}=98 \%\right)$ and $2.74 \%(95 \%$ CI: $\left.2.08-3.58, I^{2}=94 \%\right)$. Conclusion. We documented $11.07 \%$ pooled prevalence of hypothyroidism in pregnant women in India.
\end{abstract}

\section{Introduction}

Pregnancy has a significant effect on the thyroid gland and its functioning [1]. Hypothyroidism in pregnancy is defined as an increased TSH level in serum. Furthermore, based on free T4 levels, it is categorized into overt (lower free T4 levels) and subclinical hypothyroidism (normal free T4 levels) [2].

Worldwide, several studies have reported 1.5\%-4\% prevalence of hypothyroidism in pregnant women. Among them, $0.3 \%$ to $0.5 \%$ had overt hypothyroidism $(\mathrm{OH})$, and the rest had subclinical hypothyroidism (SCH) [3-5]. In India, reports on the prevalence of maternal hypothyroidism ranged between $1.2 \%$ and $67.0 \%$ in various studies $[6,7]$.

Globally, the leading cause of hypothyroidism in pregnancy is iodine deficiency, and in iodine sufficient areas, most common cause is autoimmune thyroiditis $[4,5,8]$. Other common causes are radio-iodine therapy, thyroidectomy, congenital hypothyroidism, drug use (i.e., rifampicin and phenytoin) and any hypothalamic-pituitary disease $[4,5,8]$. Women with lower thyroid reserves preconceptually are often unable to cope with increased metabolic demands during pregnancy period and can enter into the hypothyroid state. Maternal thyroid hormone levels are critical to the fetus, especially in the first trimester due to inability to produce iodothyronines before ten weeks of gestation. This is the period when neurodevelopment of fetus can potentially be hampered due to deficiency of iodothyronines [9].

In pregnant women, untreated overt hypothyroidism is associated with gestational hypertension, abruptio placenta, anemia, gestational diabetes, and postpartum hemorrhage 
[10-13]. In overt hypothyroidism, there is also an increased risk of adverse birth outcomes. Frequently associated birth outcomes are spontaneous miscarriage, low birth weight, premature birth, fetal distress, perinatal death, and stillbirth [11, 14-19]. Overt hypothyroidism also has a detrimental impact on neurocognitive development of the fetus. Subclinical hypothyroidism might also have similar adverse effects, although the evidence is inconclusive [2]. Moreover, various studies have found that children born to mother with untreated hypothyroidism are at significantly higher risk of developing neuropsychological developmental disorders, which may manifest as lower IQ scores and other learning disabilities [20-22].

Earlier studies have also proved that most of these complications can be prevented by high-risk screening pregnant women for thyroid status and providing treatment in the form of levothyroxine (LT4) [2]. To effectively start such a screening program in India, there is a need to have a national-level estimate of the prevalence of this disease.

Hence, we conducted this meta-analysis with the aim to estimate the prevalence of hypothyroidism among pregnant women in India.

\section{Methods}

This meta-analysis article is written in accordance with the PRISMA guidelines [23] and is registered in the PROSPERO database (CRD42019137955).

2.1. Eligibility Criteria. We included the studies reporting the prevalence of hypothyroidism in pregnant women in India.

Inclusion criteria: (1) community or hospital-based studies; (2) studies, providing prevalence of hypothyroidism (subclinical or overt or category nonspecified); (3) studies conducted in India; (4) type of studies: cross-sectional studies, cohort studies, or data-based analysis; (5) diagnosis based on TSH level; (6) singleton pregnancy

Exclusion criteria: (1) studies conducted in the special population groups like the diabetic mothers or mothers with pregnancy losses, etc.; (2) studies which have not reported screening methods; (3) diagnosis other than TSH levels; (4) case-control studies or experimental studies

2.2. Information Sources. We performed searches in PubMed, Web of Science, and Scopus using selected keywords. These results were supplemented by relevant studies obtained form Google Scholar and Shodhganga-Indian thesis repository (https://shodhganga.inflibnet.ac.in/). We included articles published up to December 2019. No date or language restrictions were imposed. The cross-references of the included studies were explored for additional studies. Keywords were identified with discussion among reviewers, and search query was developed separately for each database.
The main themes of search terms were thyroid (hypothyroidism OR thyroid disorder); geographic area (India); pregnancy (pregnancy OR pregnant OR antenatal OR prenatal); and prevalence (prevalence OR cross-sectional OR incidence OR cohort OR prospective OR longitudinal). To develop a robust search strategy, we used controlled descriptors (such as $\mathrm{MeSH}$ terms) and Boolean operators.

2.3. Study Selection. Two reviewers (ADG and DD) independently conducted searches on all information sources. Then, all search results were uploaded to Rayyan QCRI online software (https://rayyan.qcri.org). Rayyan QCRI helped in ensuring a systematic and comprehensive search and selection process. A third reviewer (VY) managed Rayyan QCRI software, who identified and removed the duplicate citations and ensured independent review of titles and abstracts by blinding the decisions of both reviewers.

The third reviewer also identified the discrepancies between the two reviewers and discussed them, for making consensus to select the articles. Full-text copies of all selected studies were obtained to find more details. Both reviewers (ADG and DD) reviewed the full text of articles and resolved the discrepancies by consensus. If needed, the arbitration was done by the third reviewer (VY).

We documented the reasons for the exclusion of studies explored as full text. If any study is reported as multiple publications, all publications were obtained, and data were extracted from all the publications to collect maximum relevant information. The study inclusion process is presented using the PRISMA flowchart (Figure 1). The reference management software Mendeley Desktop (https://www. mendeley.com) for Windows was used to store, organize, cite, and manage all the included articles.

2.4. Data Extraction. After selecting eligible studies, both reviewers (ADG and DD) independently performed data extraction of relevant information. Data were extracted regarding author, year of publication, study location, site (hospital- or community-based or data-based), study type, trimester, whether prepregnancy thyroid disorder patients were excluded, sample size, diagnostic criteria, and prevalence of hypothyroidism (overall, subclinical, and overt). We contacted authors of included articles for additional data whenever required. Inconsistencies in data were resolved by either consensus or seeking further information from the authors of the study. In case of disagreement between two reviewers, arbitration was done by the third reviewer (VY).

2.5. Quality Assessment of Studies. Two reviewers (ADG and DD) assessed each study's methodological quality independently using JBI critical appraisal checklist [24] for crosssectional studies. The components included in this checklist are addressing the target population, appropriateness of participant recruitment, adequacy of sample size, detailed description of study subjects, data analysis with sufficient 


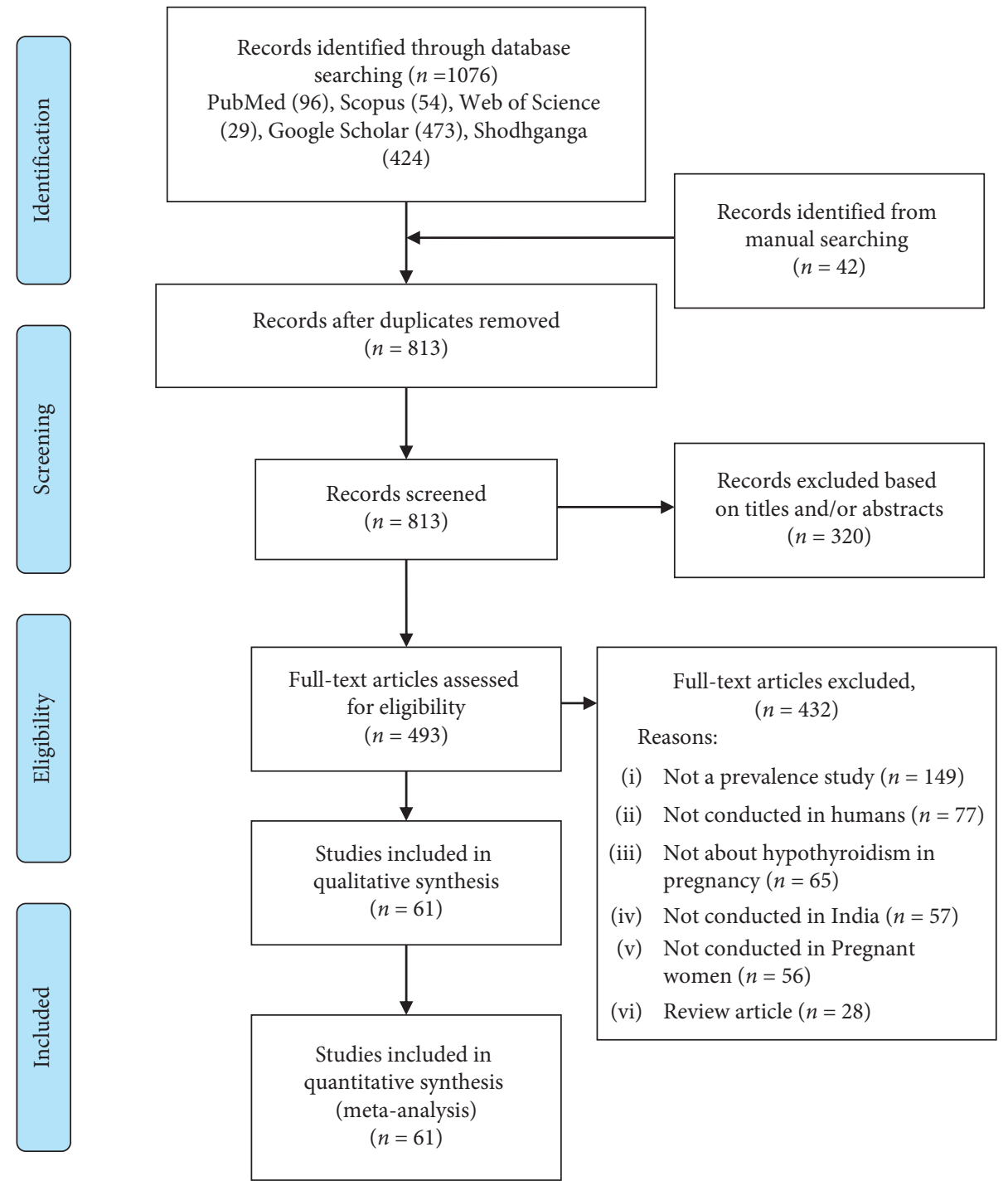

FIgURE 1: Prisma flowchart.

coverage of the identified sample, use of valid methods for identification of the condition, measurement of the condition in a standardized and reliable way for all participants, use of appropriate statistical analysis, and adequacy of response rate. For any discrepancies, the third reviewer (VY) was consulted. Studies having six or more scores (out of a total of 9) were considered optimum quality studies.

2.6. Data Synthesis and Analysis. The effect sizes were calculated for each of the included studies comprising the prevalence estimates of overall hypothyroidism, $\mathrm{OH}$, and $\mathrm{SCH}$ among pregnant women in India. Pooled estimates were calculated separately for each of them. All the analysis was done in $\mathrm{R}$ software using Meta and Metafor packages $[25,26]$.

Logit transformation (using Generalized Linear Mixed Models, GLMMs) of proportions was implemented to calculate all pooled estimates, as it is the preferred method for calculating effect size for proportions [27].
Heterogeneity between studies was examined by $I$ squared $\left(I^{2}\right)$ statistic and Cochran's $Q$ test. Due to significant heterogeneity between the studies, we used random effects models for pooling the estimates and they were reported as a proportion with $95 \%$ confidence interval. Restricted Maximum-Likelihood Estimator (REML) method was used to calculate Tau squared. Confidence intervals for individual studies were calculated using the Clopper-Pearson method. We determined the presence of publication bias by visual inspection of funnel plots and rank correlation test. A funnel plot was made between transformed proportions and standard error of transformed proportions. Statistical significance was considered as the $p$ value of less than 0.05 . Subgroup analysis of estimate of the prevalence of total hypothyroidism was done for "trimester" of participants gestational period (first/ second/third/all trimester), whether participants having prepregnancy thyroid disorder were excluded (excluded/ not excluded/not mentioned), and the setting of study (hospital-/community-/data-based). India is a federal 
union that comprises 28 states and 8 union territories. Therefore, state-wise subgroup analysis was also conducted to get state-wise estimates of the prevalence of hypothyroidism. Another subgroup analysis was conducted to analyze the difference of prevalence in studies undertaken in coastal states (Odisha, West Bengal, Gujarat, Kerala, Tamil Nadu, Andhra Pradesh, Maharashtra, Karnataka) as compared to noncoastal states (Assam, Chandigarh, Chhattisgarh, Delhi, Haryana, Jammu and Kashmir, Madhya Pradesh, Manipur, Meghalaya, Punjab, Sikkim, Telangana, Uttar Pradesh, Uttarakhand). Sensitivity analysis was done to exclude studies with a lower sample size (sample size less than 200) and to exclude studies with a quality score of less than six.

\section{Results}

On searching PubMed $(n=96)$, Scopus $(n=54)$, Web of Science $(n=29)$, Google Scholar $(n=473)$, and Shodhganga-reservoir of Indian theses $(n=424)$, a total of 1076 articles were identified related to maternal hypothyroidism.

Google Scholar uses artificial intelligence algorithms to include and rank the articles. It provides only 1000 results for each search query we make. We screened titles of all the 1000 results and found 473 relevant to our purpose.

An additional 42 articles were found eligible for inclusion through search of cross-references. After removal of 305 duplicate articles, a total of 813 articles were screened for inclusion. Based on their titles and abstract, 320 articles were excluded. Thus, the full texts of 493 articles were assessed for eligibility for the systematic review and meta-analysis. The various reasons for exclusion of studies were "not a prevalence study" ( $n=149)$, "not conducted in humans" $(n=77)$, "not about hypothyroidism in pregnancy" $(n=65)$, "not conducted in India" $(n=57)$, "not conducted in pregnant women" $(n=56)$, and "review article" $(n=28)$.

All included studies $(n=61)$ provided the prevalence of overall hypothyroidism (total population 60,066) (Figure 1) $[6,7,28-86]$. Among them, 49 studies provided the prevalence of SCH (total population 33,068), and 42 studies provided the prevalence estimates of $\mathrm{OH}$ (total population $30,980)$. Fifty-four studies were conducted in a hospitalbased setting, five were secondary data analysis, and two were community-based studies. Almost half (31 studies) of the studies were conducted in coastal states, 29 studies conducted in noncoastal states, and only 1 study conducted in multiples sites involving both coastal and noncoastal states (Table 1).

The definition of overall hypothyroidism (elevated TSH), subclinical hypothyroidism (elevated TSH with normal free thyroid hormone), and overt hypothyroidism (elevated TSH with low free thyroid hormone) was similar across the selected studies, but the reference cutoffs for TSH and free thyroid hormones $\left(\mathrm{FT}_{4}\right.$ and $\left.\mathrm{FT}_{3}\right)$ were varied across studies. Upper cutoff limit of TSH for defining hypothyroidism in pregnancy varied from 2.5 to $4.5 \mathrm{mIU} / \mathrm{L}$. For $\mathrm{OH}$, the lower cutoff limits for FT4 and FT3 varied extensively among selected studies (Table 1). Thirty-five studies used American
Thyroid Association 2011 (ATA 2011) guidelines for defining hypothyroidism [1] and the rest (26) of the studies used nonstandardized criteria for defining hypothyroidism.

3.1. Prevalence Estimates of Hypothyroidism in Pregnant Women in India. Pooled estimate (with random effects model) of the prevalence of overall hypothyroidism in pregnant women was $11.07 \%$ (95\% CI: 8.79-13.84, $\left.I^{2}=99 \%\right)$. Pooled prevalence estimates of subclinical and overt hypothyroidism were 9.51\% (95\% CI: 7.48-12.04, $\left.I^{2}=98 \%\right)$ and $2.74 \%$ (95\% CI: $\left.2.08-3.58, I^{2}=94 \%\right)$, respectively (Figures 2-4).

3.2. Subgroup Analysis of Overall Hypothyroidism Estimates. Studies that included only women with second-trimester pregnancy documented a higher prevalence of hypothyroidism (25.51, 95\% CI: 10.41-50.24) as compared to studies with women in first trimester (10.99, 95\% CI: 8.11-14.74), or any trimester of pregnancy $(10.05,95 \%$ CI: 7.33-13.65). However, these differences across various subgroups were not statistically significant $(p=0.154)$.

The estimated pooled prevalence was not statistically different among studies that excluded prepregnancy thyroid disorder patients $(11.31,95 \%$ CI: 8.57-14.79) as compared to those studies where they were included $(10.89,95 \% \mathrm{CI}$ : 7.73-15.14) in the analysis.

Studies conducted in hospital settings reported higher pooled prevalence of hypothyroidism among pregnant women (12.32, 95\% CI: 9.97-15.13) as compared to secondary data $(6.24,95 \% \mathrm{CI}: 1.83-19.17)$ and communitybased settings $(1.27,95 \% \mathrm{CI}: 0.41-3.86)(p<0.001)$.

Studies conducted in coastal states documented lower prevalence in comparison to noncoastal states though this difference was not statistically significant (coastal: 8.82, 95\% CI: 6.45-11.94 vs. non-coastal:13.49, 95\% CI: 9.77-18.34, $p=0.059$ ) (Table 2).

Studies conducted using ATA 2011 criteria reported higher prevalence (13.71, 95\% CI: 10.52-17.67) as compared to studies used other criteria (8.10, 95\% CI: 5.49-11.80) for diagnosis and this difference was statistically significant $(p=0.024)$.

State-wise pooled prevalence was also calculated and is presented in Table 3 and Figure5.

\subsection{Sensitivity Analysis of Overall Hypothyroidism Estimates.} The sensitivity analysis was conducted by removing lowerquality studies. Prevalence estimate was 10.23 (95\% CI $7.41-13.97, I^{2}: 98.4 \%, 29$ studies) after omitting studies with a quality score of less than six on JBI criteria. Another sensitivity analysis was executed to assess the effect of studies with lower sample-sizes on pooled prevalence. This analysis was performed by excluding smaller sample size studies (sample size <200) and pooled prevalence estimate was 11.22 (95\% CI: 9.00-13.91, $I^{2}$ : 98.7\%, 54 studies). Both small sample size and low-quality studies did not affect much the prevalence estimate. 
TABle 1: Summary of included studies.

\begin{tabular}{|c|c|c|c|c|c|c|}
\hline $\begin{array}{l}\text { S. } \\
\text { no }\end{array}$ & $\begin{array}{l}\text { Study (author, year of } \\
\text { publication, and study } \\
\text { location) }\end{array}$ & Setting & $\begin{array}{l}\text { Definition used ( } \Delta 1-\text { first } \\
\text { trimester, } \Delta 2 \text {-second } \\
\text { trimester, } \Delta 3 \text {-third } \\
\text { trimester) }\end{array}$ & $\begin{array}{l}\text { Hypothyroidism } \\
\text { before pregnancy }\end{array}$ & $\begin{array}{c}\text { Participant } \\
\text { characteristics } \\
(\Delta 1 \text { - first trimester, } \\
\Delta 2 \text { - second trimester, } \\
\Delta 3 \text { - third trimester })\end{array}$ & $\begin{array}{l}\text { Total } \\
\text { participants } \\
\text { number and } \\
\text { outcomes }\end{array}$ \\
\hline 1 & $\begin{array}{c}\text { Agrawal et al. 2019; New } \\
\text { Delhi [28] }\end{array}$ & Hospital-based & $\begin{array}{c}\text { SCH: TSH }>4.5 \mathrm{mIU} / \mathrm{L} \text { in } \\
\Delta 1, \Delta 2, \Delta 3 \text { with normal T4 } \\
(>8.5 \mathrm{pmol} / \mathrm{L}), \mathrm{OH}: \\
\mathrm{TSH}>4.5 \mathrm{mIU} / \mathrm{L} \text { in } \Delta 1, \Delta 2, \\
\Delta 3 \text { with low T4 }(<8.5 \mathrm{pmol} / \\
\mathrm{L})\end{array}$ & Included & $\begin{array}{c}n=250 \text {, age: not } \\
\text { mentioned, GA: } \Delta 1\end{array}$ & $\begin{array}{l}\text { TH: } 15 \\
\text { OH: } 2 \\
\text { SCH: } 13\end{array}$ \\
\hline 2 & $\begin{array}{c}\text { Diyora et al. 2019; } \\
\text { Vadodara, Gujarat [29] }\end{array}$ & Hospital-based & ATA 2011 & Excluded & $\begin{array}{c}n=200, \text { age: } \\
25.56 \pm 3.32 \text { years, GA: } \\
\Delta 1\end{array}$ & $\begin{array}{l}\text { TH: } 30 \\
\text { OH: } 9 \\
\text { SCH: } 21\end{array}$ \\
\hline 3 & $\begin{array}{l}\text { Goel et al. 2018; } \\
\text { Chandigarh [30] }\end{array}$ & Hospital-based & $\begin{array}{c}\mathrm{SCH}: \mathrm{TSH}>2.5-10 \mathrm{mIU} / \mathrm{L} \\
\text { in } \Delta 1, \Delta 2, \Delta 3, \mathrm{OH}: \\
\mathrm{TSH}>10 \mathrm{mIU} / \mathrm{L}\end{array}$ & Included & $\begin{array}{c}n=545, \text { age: } \\
26.21 \pm 3.78 \text { years, GA: } \\
\Delta 1 \Delta 2, \Delta 3\end{array}$ & $\begin{array}{l}\text { TH: } 201 \\
\text { OH: } 23 \\
\text { SCH: } 178\end{array}$ \\
\hline 4 & $\begin{array}{c}\text { Gupta et al. 2018; } \\
\text { Kanpur, Uttar Pradesh } \\
\text { [31] }\end{array}$ & Hospital-based & ATA 2011 & Included & $\begin{array}{c}n=1072 \text {, age: not } \\
\text { mentioned, GA: } \Delta 1 \Delta 2, \\
\Delta 3\end{array}$ & $\begin{array}{l}\text { TH: } 186 \\
\text { OH: } 44 \\
\text { SCH:142 }\end{array}$ \\
\hline 5 & $\begin{array}{c}\text { Hymavathi et al. 2018; } \\
\text { Nellore, Andhra Pradesh } \\
\text { [32] }\end{array}$ & Hospital-based & $\begin{array}{c}\text { SCH: TSH }>2.5 \mathrm{mIU} / \mathrm{L} \text { in } \\
\Delta 1, \mathrm{TSH}>3 \mathrm{mIU} / \mathrm{L} \text { in } \Delta 2 \\
\text { and TSH }>3.5 \mathrm{mIU} / \mathrm{L} \text { in } \Delta 3 \\
\text { with normal T4 (cutoffs not } \\
\text { mentioned); OH: } \\
\text { TSH }>2.5 \mathrm{mIU} / \mathrm{L} \text { in } \Delta 1 \text {, } \\
\mathrm{TSH}>3 \mathrm{mIU} / \mathrm{L} \text { in } \Delta 2 \text { and } \\
\mathrm{TSH}>3.5 \mathrm{mIU} / \mathrm{L} \text { in } \Delta 3 \\
\text { with low T4 (cutoffs not } \\
\text { mentioned) }\end{array}$ & Included & $\begin{array}{c}n=1000, \text { age: } 24 \text { years, } \\
\text { GA: } \Delta 1 \Delta 2, \Delta 3\end{array}$ & $\begin{array}{l}\text { TH: } 103 \\
\text { OH: } 34 \\
\text { SCH: } 69\end{array}$ \\
\hline 6 & $\begin{array}{c}\text { Kalra et al. 2018; Karnal, } \\
\text { Haryana [33] }\end{array}$ & $\begin{array}{l}\text { Secondary data } \\
\text { from hospital- } \\
\text { based study? }\end{array}$ & $\begin{array}{c}\text { Hypothyroidism: } \\
\text { TSH }>3 \mathrm{mIU} / \mathrm{L} \text { in } \Delta 1, \Delta 2, \\
\Delta 3\end{array}$ & Included & $\begin{array}{c}n=499, \text { age: not } \\
\text { mentioned, GA: } \Delta 1, \\
\Delta 2, \Delta 3\end{array}$ & TH: 11 \\
\hline 7 & $\begin{array}{c}\text { Korde et al. 2018; } \\
\text { Dabhade, Maharashtra } \\
{[34]}\end{array}$ & Hospital-based & ATA 2011 & Included & $\begin{array}{c}n=705, \text { age: not } \\
\text { mentioned, GA: } \Delta 1, \\
\Delta 2, \Delta 3\end{array}$ & $\begin{array}{l}\text { TH: } 46 \\
\text { OH: } 23 \\
\text { SCH: } 23\end{array}$ \\
\hline 8 & $\begin{array}{l}\text { Kumari et al. 2018; } \\
\text { Gorakhpur, Uttar } \\
\text { Pradesh [35] }\end{array}$ & Hospital-based & ATA 2011 & Excluded & $\begin{array}{c}n=720 \text {; age: not } \\
\text { mentioned; GA: }<20 \\
\text { week, } \Delta 1\end{array}$ & $\begin{array}{l}\text { TH: } 135 \\
\text { OH: } 20 \\
\text { SCH: } 115\end{array}$ \\
\hline 9 & $\begin{array}{c}\text { Kumari et al. 2018; } \\
\text { Visakhapatnam, Andhra } \\
\text { Pradesh [36] }\end{array}$ & Hospital-based & ATA 2011 & Excluded & $\begin{array}{c}n=4701 \text {; age: not } \\
\text { mentioned; GA: in } \Delta 1, \\
\Delta 2, \Delta 3\end{array}$ & $\begin{array}{l}\text { TH: } 880 \\
\text { OH: } 311 \\
\text { SCH:569 }\end{array}$ \\
\hline 10 & $\begin{array}{l}\text { Pahwa and Mangat 2018; } \\
\text { Amritsar, Punjab [37] }\end{array}$ & Hospital-based & ATA 2011 & Included & $\begin{array}{c}n=100 \text {; age: } 23.27 \pm 4.3 \\
\text { years; GA: } \Delta 1\end{array}$ & $\begin{array}{l}\text { TH: } 8 \\
\text { OH: } 2 \\
\text { SCH: } 6\end{array}$ \\
\hline 11 & $\begin{array}{c}\text { Panda et al. 2018; } \\
\text { Bhubaneswar, Odisha } \\
\text { [38] }\end{array}$ & Hospital-based & $\begin{array}{c}\mathrm{SCH}: \mathrm{TSH}>2.5-10 \mathrm{mIU} / \mathrm{L} \\
\text { in } \Delta 1, \Delta 2, \Delta 3 \text { with normal } \\
\text { T4 (cutoffs not } \\
\text { mentioned); OH: } \\
\text { TSH }>2.5 \mathrm{mIU} / \mathrm{L} \text { in } \Delta 1, \Delta 2 \text {, } \\
\Delta 3 \text { with low T4 (cutoffs not } \\
\text { mentioned) or } \\
\text { TSH }>10 \mathrm{mIU} / \mathrm{L}\end{array}$ & Excluded & $\begin{array}{c}n=428 \text {; age: } 23.95 \pm 3.8 \\
\text { years; GA: median } 12 \\
\text { weeks (range 6-38), } \\
\Delta 1, \Delta 2, \Delta 3\end{array}$ & $\begin{array}{l}\text { TH: } 207 \\
\text { OH: } 48 \\
\text { SCH: } 159\end{array}$ \\
\hline 12 & $\begin{array}{c}\text { Pandey et al. 2018; } \\
\text { Dehradun, Uttarakhand } \\
\text { [7] }\end{array}$ & Hospital-based & ATA 2011 & Included & $\begin{array}{c}n=100 \text {; age: } 26.6 \pm 4.13 \\
\text { years; GA: } \Delta 2\end{array}$ & $\begin{array}{l}\text { TH: } 67 \\
\text { OH: } 32 \\
\text { SCH: } 35\end{array}$ \\
\hline 13 & $\begin{array}{l}\text { Pillai and Bennet 2018; } \\
\text { Karakonam, Kerala [39] }\end{array}$ & Hospital-based & ATA 2011 & Excluded & $\begin{array}{c}n=1000 \text {; age: not } \\
\text { mentioned; GA: } \Delta 1 \\
\Delta 2, \Delta 3\end{array}$ & $\begin{array}{l}\text { TH: } 92 \\
\text { OH: } 7 \\
\text { SCH: } 85\end{array}$ \\
\hline
\end{tabular}


TABle 1: Continued.

\begin{tabular}{|c|c|c|c|c|c|c|}
\hline $\begin{array}{l}\text { S. } \\
\text { no }\end{array}$ & $\begin{array}{l}\text { Study (author, year of } \\
\text { publication, and study } \\
\text { location) }\end{array}$ & Setting & $\begin{array}{l}\text { Definition used ( } \Delta 1-\text { first } \\
\text { trimester, } \Delta 2 \text {-second } \\
\text { trimester, } \Delta 3 \text {-third } \\
\text { trimester) }\end{array}$ & $\begin{array}{l}\text { Hypothyroidism } \\
\text { before pregnancy }\end{array}$ & $\begin{array}{c}\text { Participant } \\
\text { characteristics } \\
(\Delta 1 \text {-first trimester, } \\
\Delta 2 \text {-second trimester, } \\
\Delta 3 \text { - third trimester })\end{array}$ & $\begin{array}{l}\text { Total } \\
\text { participants } \\
\text { number and } \\
\text { outcomes }\end{array}$ \\
\hline 14 & $\begin{array}{c}\text { Rohini et al. 2018; } \\
\text { Bengaluru, Karnataka } \\
{[40]}\end{array}$ & Hospital-based & ATA 2011 & Excluded & $\begin{array}{c}n=810 ; \text { age: } 27.2 \text { years; } \\
\text { GA: } \Delta 1, \Delta 2, \Delta 3\end{array}$ & $\begin{array}{l}\text { TH: } 78 \\
\text { OH: } \\
\text { SCH: }\end{array}$ \\
\hline 15 & $\begin{array}{l}\text { Devi and Vanaja 2017; } \\
\text { Visakhapatnam, Andhra } \\
\text { Pradesh [41] }\end{array}$ & Hospital-based & $\begin{array}{l}\text { SCH: TSH } 3-10 \mathrm{mIU} / \mathrm{L} \text { in } \\
\Delta 1, \Delta 2, \Delta 3 \text { with normal T4 } \\
\text { (cutoffs not mentioned); } \\
\text { OH: TSH }>3 \mathrm{mIU} / \mathrm{L} \text { in } \Delta 1 \text {, } \\
\Delta 2, \Delta 3 \text { with low T4 (cutoffs } \\
\text { not mentioned) or } \\
\text { TSH }>10 \mathrm{mIU} / \mathrm{L}\end{array}$ & Excluded & $\begin{array}{c}n=1000 \text {; age: not } \\
\text { mentioned; GA: }<12 \\
\text { week, } \Delta 1\end{array}$ & $\begin{array}{l}\text { TH: } 68 \\
\text { OH: } 17 \\
\text { SCH: } 51\end{array}$ \\
\hline 16 & $\begin{array}{c}\text { Dubey and Pradhan } \\
\text { 2017; Gangtok, Sikkim } \\
\text { [42] }\end{array}$ & Hospital-based & ATA 2011 & Excluded & $\begin{array}{c}n=200 \text {; age: } 20-35 \\
\text { years; GA: 6-24 weeks, } \\
\qquad 1, \Delta 2, \Delta 3\end{array}$ & $\begin{array}{l}\text { TH: } 20 \\
\text { OH: } 4 \\
\text { SCH: } 16\end{array}$ \\
\hline 17 & $\begin{array}{c}\text { Gedam and Rajput 2017; } \\
\text { Mumbai, Maharashtra } \\
{[43]}\end{array}$ & Hospital-based & ATA 2011 & Excluded & $\begin{array}{c}n=350 \text {; age: not } \\
\text { mentioned; GA: } \Delta 1, \\
\Delta 2, \Delta 3\end{array}$ & $\begin{array}{l}\text { TH: } 41 \\
\text { OH: } 14 \\
\text { SCH: } 27\end{array}$ \\
\hline 18 & $\begin{array}{c}\text { Gupta et al. 2017; Patiala, } \\
\text { Punjab }[44]\end{array}$ & Hospital-based & $\begin{array}{c}\text { SCH: TSH }>3-6 \mathrm{mIU} / \mathrm{L} \text { in } \\
\Delta 1, \Delta 2, \Delta 3 ; \text { OH (clinical } \\
\text { hypothyroidism): } \\
\text { TSH }>6 \text { mIU/L in } \Delta 1, \Delta 2, \\
\Delta 3\end{array}$ & Included & $\begin{array}{c}n=2122 \text {; age: not } \\
\text { mentioned; GA: } \Delta 1, \\
\Delta 2, \Delta 3\end{array}$ & $\begin{array}{l}\text { TH: } 132 \\
\text { OH: } 52 \\
\text { SCH: } 80\end{array}$ \\
\hline 19 & $\begin{array}{l}\text { Sapna and Mehazeena } \\
\text { 2017; Mumbai, } \\
\text { Maharashtra [45] }\end{array}$ & Secondary data & $\begin{array}{c}\text { Hypothyroidism: } \\
\text { TSH }>3 \mathrm{mIU} / \mathrm{L} \text { in } \Delta 1, \Delta 2, \\
\Delta 3\end{array}$ & Included & $\begin{array}{c}n=12300 \text {; age: not } \\
\text { mentioned; GA: } \Delta 1 \text {, } \\
\Delta 2, \Delta 3\end{array}$ & $\begin{array}{l}\text { TH: } 150 \\
\text { OH: } \\
\text { SCH: }\end{array}$ \\
\hline 20 & $\begin{array}{c}\text { Indira et al. 2017; } \\
\text { Vizianagaram, Andhra } \\
\text { Pradesh [46] }\end{array}$ & Hospital-based & ATA 2011 & Included & $\begin{array}{c}n=333 \text {; age: not } \\
\text { mentioned; GA: } \Delta 1 \\
\Delta 2, \Delta 3\end{array}$ & $\begin{array}{l}\text { TH: } 46 \\
\text { OH: } 1 \\
\text { SCH: } 45\end{array}$ \\
\hline 21 & $\begin{array}{c}\text { Pokhanna et al. 2017; } \\
\text { Indore, Madhya Pradesh } \\
\text { [47] }\end{array}$ & Hospital-based & ATA 2011 & Excluded & $\begin{array}{c}n=300 \text {; age: not } \\
\text { mentioned; GA: } 13-26 \\
\text { weeks, } \Delta 2\end{array}$ & $\begin{array}{l}\text { TH: } 39 \\
\text { OH: } 9 \\
\text { SCH: } 30\end{array}$ \\
\hline 22 & $\begin{array}{c}\text { Sannaboraiah et al. 2017; } \\
\text { not provided, Karnataka } \\
{[48]}\end{array}$ & Hospital-based & $\begin{array}{c}\text { SCH: TSH }>2.5 \mathrm{mIU} / \mathrm{L} \text { in } \\
\Delta 1 \text { and }>3 \mathrm{mIU} / \mathrm{L} \text { in } \Delta 2 \\
\text { and }>3.5 \mathrm{mIU} / \mathrm{L} \text { in } \Delta 3 \text { with } \\
\text { normal T3, T4 (cutoffs not } \\
\text { mentioned) }\end{array}$ & Excluded & $\begin{array}{c}n=200 \text {; age: not } \\
\text { mentioned; GA: } \Delta 1, \\
\Delta 2, \Delta 3\end{array}$ & $\begin{array}{l}\text { TH: } 19 \\
\text { SCH: } 19\end{array}$ \\
\hline 23 & $\begin{array}{c}\text { Thenmozhi 2017; } \\
\text { Kancheepuram, Tamil } \\
\text { Nadu [49] }\end{array}$ & Hospital-based & ATA 2011 & Included & $\begin{array}{c}n=1000 \text {; age: not } \\
\text { mentioned; GA: } \Delta 1, \\
\Delta 2, \Delta 3\end{array}$ & $\begin{array}{l}\text { TH: } 31 \\
\text { OH: } 6 \\
\text { SCH: } 25\end{array}$ \\
\hline 24 & $\begin{array}{c}\text { Wagh et al. 2017; } \\
\text { Nagpur, Maharashtra } \\
{[50]}\end{array}$ & Secondary data & ATA 2011 & Included & $\begin{array}{c}n=400 \text {; age: not } \\
\text { mentioned; GA: } \Delta 1, \\
\Delta 2, \Delta 3\end{array}$ & $\begin{array}{l}\text { TH: } 136 \\
\text { OH: } 16 \\
\text { SCH: } 120\end{array}$ \\
\hline 25 & $\begin{array}{c}\text { Anitha and Rao 2016; } \\
\text { Hyderabad, Telangana } \\
\text { [51] }\end{array}$ & Hospital-based & ATA 2011 & Included & $\begin{array}{c}n=1062 \text {; age: not } \\
\text { mentioned; GA: } \Delta 1, \\
\Delta 2, \Delta 3\end{array}$ & $\begin{array}{l}\text { TH: } 215 \\
\text { OH: } 66 \\
\text { SCH: } 149\end{array}$ \\
\hline 26 & $\begin{array}{c}\text { Chunchaiah et al. 2016; } \\
\text { Banguluru, Karnataka } \\
\text { [52] }\end{array}$ & Hospital-based & ATA 2011 & Included & $\begin{array}{c}n=800 \text {; age: not } \\
\text { mentioned; GA: } \Delta 1, \\
\Delta 2, \Delta 3\end{array}$ & $\begin{array}{l}\text { TH: } 81 \\
\text { OH: } 22 \\
\text { SCH: } 59\end{array}$ \\
\hline
\end{tabular}


TABle 1: Continued.

\begin{tabular}{|c|c|c|c|c|c|c|}
\hline $\begin{array}{l}\text { S. } \\
\text { no }\end{array}$ & $\begin{array}{l}\text { Study (author, year of } \\
\text { publication, and study } \\
\text { location) }\end{array}$ & Setting & $\begin{array}{l}\text { Definition used ( } \Delta 1-\text { first } \\
\text { trimester, } \Delta 2 \text {-second } \\
\text { trimester, } \Delta 3 \text {-third } \\
\text { trimester) }\end{array}$ & $\begin{array}{l}\text { Hypothyroidism } \\
\text { before pregnancy }\end{array}$ & $\begin{array}{c}\text { Participant } \\
\text { characteristics } \\
(\Delta 1 \text {-first trimester, } \\
\Delta 2 \text { - second trimester, } \\
\Delta 3 \text { - third trimester })\end{array}$ & $\begin{array}{l}\text { Total } \\
\text { participants } \\
\text { number and } \\
\text { outcomes }\end{array}$ \\
\hline 27 & $\begin{array}{l}\text { Dhanwal et al. 2016; } \\
\text { Allahabad, Bengaluru, } \\
\text { Chennai, Kolkata, } \\
\text { Hyderabad, Nasik, } \\
\text { Rohtak, Pune, New } \\
\text { Delhi, Srinagar, and } \\
\text { Vizag,Uttar Pradesh, } \\
\text { Karnataka, Tamil Nadu, } \\
\text { West Bengal, } \\
\text { Maharashtra, Haryana, } \\
\text { Maharashtra, Delhi, } \\
\text { Kashmir and Andhra } \\
\text { Pradesh [53] }\end{array}$ & Hospital-based & $\begin{array}{c}\text { Hypothyroidism: } \\
\mathrm{TSH}>4.5 \mathrm{mIU} / \mathrm{L} \text { in } \Delta 1, \Delta 2, \\
\Delta 3\end{array}$ & Included & $\begin{array}{c}n=2599 \text {; age: } 25.5 \pm 5.6 \\
\text { years; GA: } 19.3 \pm 15.9 \\
\text { weeks, } \Delta 1, \Delta 2, \Delta 3\end{array}$ & $\begin{array}{l}\text { TH: } 816 \\
\text { OH: } \\
\text { SCH: }\end{array}$ \\
\hline 28 & $\begin{array}{c}\text { Hassan et al. 2016; J \& K, } \\
\text { Srinagar [54] }\end{array}$ & Hospital-based & ATA 2011 & Included & $\begin{array}{c}n=342 \text {; age: not } \\
\text { mentioned; GA: } \Delta 1, \\
\Delta 2, \Delta 3\end{array}$ & $\begin{array}{l}\text { TH: } 117 \\
\text { OH: } 3 \\
\text { SCH: } 114\end{array}$ \\
\hline 29 & $\begin{array}{c}\text { Mandal et al. } 2016 \text {; } \\
\text { Kolkata, West Bengal } \\
{[55]}\end{array}$ & Hospital-based & ATA 2011 & Excluded & $\begin{array}{c}n=510 \text {; age: } 18.7 \pm 3.52 \\
\text { years; GA: } 7.6 \pm 1.12 \\
\text { weeks, } \Delta 1\end{array}$ & $\begin{array}{l}\text { TH: } 168 \\
\text { OH: } \\
\text { SCH:168 }\end{array}$ \\
\hline 30 & $\begin{array}{c}\text { Faheema Farvin } 2016 \text {; } \\
\text { Chennai, Tamil Nadu } \\
\text { [56] }\end{array}$ & Hospital-based & $\begin{array}{c}\text { SCH: TSH }>3 \mathrm{mIU} / \mathrm{L} \text { in } \\
\Delta 1, \Delta 2, \Delta 3 \text { with normal T4} \\
(11.84 \pm 3.86 \mathrm{pmol} / \mathrm{L}) ; \mathrm{OH}: \\
\mathrm{TSH}>3 \mathrm{mIU} / \mathrm{L} \text { in } \Delta 1, \Delta 2 \\
\Delta 3 \text { with low } \mathrm{T} 4 \\
(<11.84 \mathrm{pmol} / \mathrm{L}) \text { or } \\
\text { TSH }>10 \mathrm{mIU} / \mathrm{L}\end{array}$ & Excluded & $\begin{array}{l}n=217 \text {; age: } 27.5 \pm 2.12 \\
\text { years; GA: } \Delta 1, \Delta 2, \Delta 3\end{array}$ & $\begin{array}{l}\text { TH: } 26 \\
\text { OH: } \\
\text { SCH: } 26\end{array}$ \\
\hline 31 & $\begin{array}{c}\text { Prasad et al. 2016; } \\
\text { Trivandrum, Kerala [57] }\end{array}$ & Hospital-based & ATA 2011 & Included & $\begin{array}{c}n=500 ; \text { age: } 25.4 \pm 3.7 \\
\text { years; GA: } \Delta 1\end{array}$ & $\begin{array}{l}\text { TH: } 28 \\
\text { OH: } 8 \\
\text { SCH: } 20\end{array}$ \\
\hline 32 & $\begin{array}{c}\text { Rao and Patibandla 2016; } \\
\text { Hyderabad, Telangana } \\
\text { [58] }\end{array}$ & Secondary data & ATA 2011 & Included & $\begin{array}{c}n=1062 ; \text { age: } 22.1 \\
\text { years; GA: } \Delta 1, \Delta 2, \Delta 3\end{array}$ & $\begin{array}{l}\text { TH: } 215 \\
\text { OH: } 66 \\
\text { SCH: } 149\end{array}$ \\
\hline 33 & $\begin{array}{c}\text { Saraladevi et al. 2016; } \\
\text { Warangal, Maharashtra } \\
\text { [59] }\end{array}$ & Hospital-based & ATA 2011 & Included & $\begin{array}{l}n=1000 \text {; age: not } \\
\text { mentioned; GA: } \Delta 1\end{array}$ & $\begin{array}{l}\text { TH: } 92 \\
\text { OH: } 28 \\
\text { SCH: } 64\end{array}$ \\
\hline 34 & $\begin{array}{c}\text { Singla et al. 2016; } \\
\text { Ludhiana, Punjab [60] }\end{array}$ & Secondary data & ATA 2011 & Included & $\begin{array}{c}n=5400 \text {; age: not } \\
\text { mentioned; GA: } \Delta 1, \\
\Delta 2, \Delta 3\end{array}$ & $\begin{array}{l}\text { TH: } 189 \\
\text { OH: } \\
\text { SCH: }\end{array}$ \\
\hline 35 & $\begin{array}{c}\text { Stella and Minnalkodi } \\
\text { 2016; Chennai, Tamil } \\
\text { Nadu [61] }\end{array}$ & Hospital-based & ATA 2011 & Included & $\begin{array}{c}n=1000 \text {; age: not } \\
\text { mentioned; GA: } \Delta 1\end{array}$ & $\begin{array}{l}\text { TH: } 31 \\
\text { OH: } 6 \\
\text { SCH: } 25\end{array}$ \\
\hline 36 & $\begin{array}{l}\text { Tiwari et al. 2016; New } \\
\text { Delhi, Delhi [62] }\end{array}$ & Hospital-based & ATA 2011 & Excluded & $\begin{array}{c}n=967 ; \text { age: not } \\
\text { mentioned; GA: } \Delta 1 \\
\Delta 2, \Delta 3\end{array}$ & $\begin{array}{l}\text { TH: } 69 \\
\text { OH: } 21 \\
\text { SCH: } 48\end{array}$ \\
\hline 37 & $\begin{array}{l}\text { Chauhan et al. 2015; } \\
\text { Jabalpur, Madhya } \\
\text { Pradesh [63] }\end{array}$ & Hospital-based & ATA 2011 & Included & $\begin{array}{c}n=250 \text {; age: majority } \\
20-30 \text { years; GA: } \Delta 1, \\
\Delta 2, \Delta 3\end{array}$ & $\begin{array}{l}\text { TH: } 59 \\
\text { OH: } 5 \\
\text { SCH: } 54\end{array}$ \\
\hline 38 & $\begin{array}{l}\text { George et al. 2015; Kochi, } \\
\text { Kerala [64] }\end{array}$ & Hospital-based & ATA 2011 & Included & $\begin{array}{c}n=1575 \text {; age: not } \\
\text { mentioned; GA: } \Delta 1 \text {, } \\
\Delta 2, \Delta 3\end{array}$ & $\begin{array}{l}\text { TH: } 100 \\
\text { OH: } \\
\text { SCH: }\end{array}$ \\
\hline 39 & $\begin{array}{c}\text { Kishore et al. 2015; } \\
\text { Raipur, Chhattisgarh } \\
{[65]}\end{array}$ & Hospital-based & ATA 2011 & Included & $\begin{array}{l}n=263 \text {; age: not } \\
\text { mentioned; GA: } \Delta 1\end{array}$ & $\begin{array}{l}\text { TH: } 25 \\
\text { OH: } 9 \\
\text { SCH: } 16\end{array}$ \\
\hline 40 & $\begin{array}{c}\text { Mohammed and } \\
\text { Chandrashekar 2015; } \\
\text { Bellary, Karnataka [66] }\end{array}$ & Hospital-based & ATA 2011 & Included & $\begin{array}{c}n=787 \text {; age: not } \\
\text { mentioned; GA: } \Delta 1 \\
\Delta 2, \Delta 3\end{array}$ & $\begin{array}{l}\text { TH: } 50 \\
\text { OH: } \\
\text { SCH: }\end{array}$ \\
\hline
\end{tabular}


TABle 1: Continued.

\begin{tabular}{|c|c|c|c|c|c|c|}
\hline $\begin{array}{l}\text { S. } \\
\text { no }\end{array}$ & $\begin{array}{l}\text { Study (author, year of } \\
\text { publication, and study } \\
\text { location) }\end{array}$ & Setting & $\begin{array}{l}\text { Definition used ( } \Delta 1 \text {-first } \\
\text { trimester, } \Delta 2 \text {-second } \\
\text { trimester, } \Delta 3 \text {-third } \\
\text { trimester) }\end{array}$ & $\begin{array}{l}\text { Hypothyroidism } \\
\text { before pregnancy }\end{array}$ & $\begin{array}{c}\text { Participant } \\
\text { characteristics } \\
(\Delta 1 \text {-first trimester, } \\
\Delta 2 \text { - second trimester, } \\
\Delta 3 \text { - third trimester })\end{array}$ & $\begin{array}{l}\text { Total } \\
\text { participants } \\
\text { number and } \\
\text { outcomes }\end{array}$ \\
\hline 41 & $\begin{array}{c}\text { Neeliyara and Kumari } \\
\text { 2015; Alleppey, Kerala } \\
\text { [6] }\end{array}$ & $\begin{array}{l}\text { Community- } \\
\text { based }\end{array}$ & $\begin{array}{c}\text { SCH: TSH }>4.5 \mathrm{mIU} / \mathrm{L} \text { in } \\
\Delta 1, \Delta 2, \Delta 3 \text { with normal T4 } \\
(>0.62 \mathrm{ng} / \mathrm{dl}) ; \mathrm{OH}: \\
\mathrm{TSH}>4.5 \mathrm{mIU} / \mathrm{L} \text { in } \Delta 1, \Delta 2 \\
\Delta 3 \text { with low T4 }(<0.62 \mathrm{ng} / \\
\text { dl })\end{array}$ & Included & $\begin{array}{c}n=166 \text {; age: not } \\
\text { mentioned; GA: } \Delta 1, \\
\Delta 2, \Delta 3\end{array}$ & $\begin{array}{l}\text { TH: } 2 \\
\text { OH: } 0 \\
\text { SCH: } 2\end{array}$ \\
\hline 42 & $\begin{array}{c}\text { Padmavathi and } \\
\text { Prasanna 2015; } \\
\text { Visakhapatnam, Andhra } \\
\text { Pradesh [67] }\end{array}$ & Hospital-based & $\begin{array}{c}\text { Hypothyroidism: TSH } \\
\text { more than } 2.3 \mathrm{mIU} / \mathrm{L} / \mathrm{L} \text {, } \\
3.7 \mathrm{mIU} / \mathrm{L}, 3.4 \mathrm{mIU} / \mathrm{L} \text { in } \\
\Delta 1, \Delta 2 \text {, and } \Delta 3 \\
\text { respectively }\end{array}$ & Excluded & $\begin{array}{c}n=1000 ; \text { age: not } \\
\text { mentioned; GA: }<12 \\
\text { week, } \Delta 1\end{array}$ & $\begin{array}{l}\text { TH: } 66 \\
\text { OH: } \\
\text { SCH: }\end{array}$ \\
\hline 43 & $\begin{array}{l}\text { Rajput et al. 2015; } \\
\text { Rohtak, Haryana [68] }\end{array}$ & Hospital-based & ATA 2011 & Excluded & $\begin{array}{c}n=461 ; \text { age: } \\
23.79 \pm 3.47 \text { years; GA: } \\
8 \text { weeks } 5 \text { days., } \Delta 1\end{array}$ & $\begin{array}{l}\text { TH: } 105 \\
\text { OH: } 6 \\
\text { SCH: } 99\end{array}$ \\
\hline 44 & $\begin{array}{l}\text { Singh et al. 2015; Imphal, } \\
\text { Manipur [69] }\end{array}$ & Hospital-based & $\begin{array}{c}\mathrm{SCH}: \mathrm{TSH}>3 \mathrm{mIU} / \mathrm{L} \text { in } \\
\Delta 1, \Delta 2, \Delta 3 \text { with normal T4 } \\
(>7.5 \mathrm{mcg} / \mathrm{dl}) ; \mathrm{OH}: \\
\mathrm{TSH}>3 \mathrm{mIU} / \mathrm{L} \text { in } \Delta 1, \Delta 2, \\
\Delta 3 \text { with Low T4 }(<7.5 \mathrm{mcg} / \\
\mathrm{dl})\end{array}$ & Excluded & $\begin{array}{l}n=400 \text {; age: } 26.8 \pm 8.2 \\
\text { years; GA: } \Delta 1, \Delta 2, \Delta 3\end{array}$ & $\begin{array}{l}\text { TH: } 90 \\
\text { OH: } 18 \\
\text { SCH: } 72\end{array}$ \\
\hline 45 & $\begin{array}{l}\text { Ajmani et al. 2014; Delhi, } \\
\text { Delhi [70] }\end{array}$ & Hospital-based & $\begin{array}{c}\text { SCH: TSH }>3 \mathrm{mIU} / \mathrm{L} \text { in } \\
\Delta 1, \Delta 2, \Delta 3 \text { with normal T4 } \\
(0.8-2 \mathrm{ng} / \mathrm{dl}) ; \mathrm{OH}: \\
\mathrm{TSH}>3 \mathrm{mIU} / \mathrm{L} \text { with low } \\
\mathrm{T} 4(<0.8 \mathrm{ng} / \mathrm{dl})\end{array}$ & Excluded & $\begin{array}{c}n=400 \text {; age: not } \\
\text { mentioned; GA: } 13-26 \\
\text { weeks, } \Delta 1\end{array}$ & $\begin{array}{l}\text { TH: } 48 \\
\text { OH: } 12 \\
\text { SCH: } 36\end{array}$ \\
\hline 46 & $\begin{array}{c}\text { Das et al. 2014; Joti Gaon, } \\
\text { Assam [71] }\end{array}$ & Hospital-based & ATA 2011 & Included & $\begin{array}{c}n=499 ; \text { age: } 18-35 \\
\text { years; GA: } \Delta 1\end{array}$ & $\begin{array}{l}\text { TH: } 218 \\
\text { OH: } \\
\text { SCH: }\end{array}$ \\
\hline 47 & $\begin{array}{l}\text { Dave et al. 2014; Indore, } \\
\text { Madhya Pradesh [72] }\end{array}$ & Hospital-based & ATA 2011 & Included & $\begin{array}{c}n=305 \text {; age: } 24.46(2) \\
\text { years; GA: } 9.09 \text { weeks, } \\
\Delta 1\end{array}$ & $\begin{array}{l}\text { TH: } 31 \\
\text { OH: } 30 \\
\text { SCH: } 1\end{array}$ \\
\hline 48 & $\begin{array}{l}\text { Jaiswal et al. 2014; } \\
\text { Banglore, Karnataka [73] }\end{array}$ & Hospital-based & ATA 2011 & Excluded & $\begin{array}{c}n=334 \text {; age: not } \\
\text { mentioned; GA: } \\
10.3 \pm 2.5 \text { weeks, } \Delta 1\end{array}$ & $\begin{array}{l}\text { TH: } 42 \\
\text { OH: } 12 \\
\text { SCH: } 30\end{array}$ \\
\hline 49 & $\begin{array}{l}\text { Nabhi and Bhashyakarla } \\
\text { 2014; Hyderabad, } \\
\text { Telangana [74] }\end{array}$ & Hospital-based & ATA 2011 & Included & $\begin{array}{c}n=322 \text {; age: not } \\
\text { mentioned; GA: } \Delta 2\end{array}$ & $\begin{array}{l}\text { TH: } 89 \\
\text { OH: } 13 \\
\text { SCH: } 76\end{array}$ \\
\hline 50 & $\begin{array}{c}\text { Bandela et al. 2013; } \\
\text { Nandyal, Andhra } \\
\text { Pradesh [75] }\end{array}$ & Hospital-based & $\begin{array}{l}\text { SCH: TSH } 4-10 \mathrm{mIU} / \mathrm{L} \text { in } \\
\Delta 1, \Delta 2, \Delta 3 \text { with normal T4 } \\
\text { (cutoffs not mentioned); } \\
\text { OH: TSH } 4-10 \mathrm{mIU} / \mathrm{L} \text { in } \\
\Delta 1, \Delta 2, \Delta 3 \text { with low T4 } \\
\text { (cutoffs not mentioned) or } \\
\text { TSH }>10 \mathrm{mIU} / \mathrm{L}\end{array}$ & Included & $\begin{array}{c}n=139 \text {; age: median } 25 \\
\text { years, } 17-35 \text { years; GA: } \\
\text { only <13 week } \\
\text { included, median } 8.5 \\
\text { weeks, } \Delta 1\end{array}$ & $\begin{array}{l}\text { TH: } 18 \\
\text { OH: } 4 \\
\text { SCH: } 14\end{array}$ \\
\hline 51 & $\begin{array}{c}\text { Dhanwal et al. 2013; New } \\
\text { Delhi, Delhi [76] }\end{array}$ & Hospital-based & $\begin{array}{c}\mathrm{SCH}: \mathrm{TSH}>4.5 \mathrm{mIU} / \mathrm{L} \text { in } \\
\Delta 1, \Delta 2, \Delta 3 \text { with normal T3, } \\
\text { T4 (cutoffs not } \\
\text { mentioned); OH: } \\
\mathrm{TSH}>4.5 \mathrm{mIU} / \mathrm{L} \text { with low } \\
\text { T3, T4 (cut-offs not } \\
\text { mentioned); } \\
\text { thyrotoxicosis: } \\
\text { TSH }>150 \mathrm{mIU} / \mathrm{L}\end{array}$ & Included & $\begin{array}{c}n=1000 ; \text { age: } \\
25.6 \pm 11.1 \text { years; GA: } \\
9.2 \pm 3.4 \text { weeks, } \Delta 1\end{array}$ & $\begin{array}{l}\text { TH: } 142 \\
\text { OH: } 7 \\
\text { SCH: } 135\end{array}$ \\
\hline
\end{tabular}


TABle 1: Continued.

\begin{tabular}{|c|c|c|c|c|c|c|}
\hline $\begin{array}{l}\text { S. } \\
\text { no }\end{array}$ & $\begin{array}{l}\text { Study (author, year of } \\
\text { publication, and study } \\
\text { location) }\end{array}$ & Setting & $\begin{array}{l}\text { Definition used ( } \Delta 1-\text { first } \\
\text { trimester, } \Delta 2 \text {-second } \\
\text { trimester, } \Delta 3 \text {-third } \\
\text { trimester) }\end{array}$ & $\begin{array}{l}\text { Hypothyroidism } \\
\text { before pregnancy }\end{array}$ & $\begin{array}{c}\text { Participant } \\
\text { characteristics } \\
(\Delta 1 \text {-first trimester, } \\
\Delta 2 \text { - second trimester, } \\
\Delta 3 \text { - third trimester })\end{array}$ & $\begin{array}{l}\text { Total } \\
\text { participants } \\
\text { number and } \\
\text { outcomes }\end{array}$ \\
\hline 52 & $\begin{array}{l}\text { Konin and Bhinder 2013; } \\
\text { Gulbarga, Karnataka [77] }\end{array}$ & Hospital-based & $\begin{array}{c}\text { SCH: TSH }>4.2 \mathrm{mIU} / \mathrm{L} \text { in } \\
\Delta 1, \Delta 2, \Delta 3 \text { with normal } \\
\text { FT4 }(0.80-2.7 \mathrm{ng} / \mathrm{dl}) \text { and } \\
\text { FT3 }(2-3.8 \mathrm{pg} / \mathrm{mL}) ; \mathrm{OH}: \\
\text { TSH }>4.2 \mathrm{mIU} / \mathrm{L} \text { with low } \\
\text { FT4 }(<0.80 \mathrm{ng} / \mathrm{dl}) \text { and FT3 } \\
(<2 \mathrm{pg} / \mathrm{mL})\end{array}$ & Excluded & $\begin{array}{c}n=400 ; \text { age: not } \\
\text { mentioned; GA: } \leq 14 \\
\text { weeks, } \Delta 1\end{array}$ & $\begin{array}{l}\text { TH: } 32 \\
\text { OH: } 0 \\
\text { SCH: } 32\end{array}$ \\
\hline 53 & $\begin{array}{c}\text { Pradhan et al. 2013; } \\
\text { Lucknow, Uttar Pradesh } \\
\text { [78] }\end{array}$ & Hospital-based & $\begin{array}{c}\mathrm{SCH}: \mathrm{TSH}>2.5 \mathrm{mIU} / \mathrm{L} \text { in } \\
\Delta 1, \Delta 2, \Delta 3 \text { with normal T3, } \\
\text { T4 (cutoffs not } \\
\text { mentioned); OH: } \\
\mathrm{TSH}>2.5 \mathrm{mIU} / \mathrm{L} \text { with low } \\
\text { T3, T4 (cut-offs not } \\
\text { mentioned) }\end{array}$ & Included & $\begin{array}{c}n=2479 \text {; age: mostly } \\
20-40,2<20 \mathrm{yr}, 2>40 \\
\text { years; GA: } \Delta 1, \Delta 2, \Delta 3\end{array}$ & $\begin{array}{l}\text { TH: } 196 \\
\text { OH: } 29 \\
\text { SCH: } 167\end{array}$ \\
\hline 54 & $\begin{array}{l}\text { Boyapati and Magshetty } \\
\text { 2012; Gulbarga, } \\
\text { Karnataka [79] }\end{array}$ & Hospital-based & $\begin{array}{c}\text { SCH: TSH }>5 \mathrm{mIU} / \mathrm{L} \text { in } \\
\Delta 1, \Delta 2, \Delta 3 \text { with normal T4 } \\
(0.93-1.7 \mathrm{ng} / \mathrm{dl}) ; \mathrm{OH}: \\
\mathrm{TSH}>4.5 \mathrm{mIU} / \mathrm{L} \text { in } \Delta 1, \Delta 2 \\
\Delta 3 \text { with low T4 }(<0.93 \mathrm{ng} / \\
\mathrm{dl})\end{array}$ & Excluded & $\begin{array}{c}n=100 \text {; age: not } \\
\text { mentioned; GA: } \Delta 1, \\
\Delta 2, \Delta 3\end{array}$ & $\begin{array}{l}\text { TH: } 3 \\
\text { OH: } \\
\text { SCH: } 3\end{array}$ \\
\hline 55 & $\begin{array}{c}\text { Goel et al. 2012; } \\
\text { Chandigarh, Chandigarh } \\
{[80]}\end{array}$ & Hospital-based & $\begin{array}{c}\text { Hypothyroidism: } \\
\mathrm{TSH}>5.5 \mathrm{mIU} / \mathrm{L} \text { in } \Delta 1, \Delta 2, \\
\Delta 3\end{array}$ & Excluded & $\begin{array}{c}n=976 \text {; age: known } \\
\text { hyothyroid: } 27.6 \pm 2.9 \\
\text { new hypothyroid: } \\
27.5 \pm 4.1, \text { euthyroid } \\
\text { group: } 25.5 \pm 3.9 ; \mathrm{GA} \text { : } \\
\text { known hyothyroid: } \\
19.5 \pm 9.4, \text { new } \\
\text { hypothyroid: } \\
20.3 \pm 8.9, \text { euthyroid } \\
\text { group: } 19.6 \pm 7.8, \Delta 1 \\
\Delta 2, \Delta 3\end{array}$ & $\begin{array}{l}\text { TH: } 37 \\
\text { OH: } \\
\text { SCH: }\end{array}$ \\
\hline 56 & $\begin{array}{c}\text { Rana 2012; Vadodara, } \\
\text { Gujarat }[81]\end{array}$ & Hospital-based & ATA 2011 & Included & $\begin{array}{c}n=194 \text {; age: } 23.3(3.6) \\
\text { years and; GA: } \Delta 1, \Delta 2, \\
\Delta 3\end{array}$ & $\begin{array}{l}\text { TH: } 54 \\
\text { OH: } 13 \\
\text { SCH: } 41\end{array}$ \\
\hline 57 & $\begin{array}{l}\text { Nambiar et al. 2011; } \\
\text { Mumbai, Maharashtra } \\
{[82]}\end{array}$ & Hospital-based & $\begin{array}{c}\text { Hypothyroidism: } \\
\text { TSH }>4 \mathrm{mIU} / \mathrm{L} \text { in } \Delta 1, \Delta 2, \\
\Delta 3 \\
\text { SCH: } \mathrm{TSH}>5.5 \mathrm{mIU} / \mathrm{L} \text { in }\end{array}$ & Excluded & $\begin{array}{c}n=483 \text {; age: } \\
25.19 \pm 4.17 \text { years; GA: } \\
10.03 \pm 1.87 \text { weeks, } \Delta 1\end{array}$ & $\begin{array}{l}\text { TH: } 24 \\
\text { OH: } \\
\text { SCH: }\end{array}$ \\
\hline 58 & $\begin{array}{c}\text { Sahu et al. 2010; } \\
\text { Lucknow and New Delhi, } \\
\text { Uttar Pradesh and New } \\
\text { Delhi [83] }\end{array}$ & Hospital-based & $\begin{array}{c}\Delta 1, \Delta 2, \Delta 3 \text { with normal T3, } \\
\text { T4 (cutoffs not } \\
\text { mentioned); OH: } \\
\text { TSH }>5.5 \text { mIU/L with low } \\
\text { T3, T4 (cutoffs not } \\
\text { mentioned) }\end{array}$ & Included & $\begin{array}{c}n=633 \text {; age: not } \\
\text { mentioned; GA: } 13-26 \\
\text { weeks, } \Delta 2\end{array}$ & $\begin{array}{l}\text { TH: } 70 \\
\text { OH: } 29 \\
\text { SCH: } 41\end{array}$ \\
\hline 59 & $\begin{array}{c}\text { Gayathri et al. 2009; } \\
\text { Chennai, Tamil Nadu } \\
\text { [84] }\end{array}$ & Hospital-based & $\begin{array}{c}\mathrm{SCH}: \mathrm{TSH}>5 \mathrm{mIU} / \mathrm{L} \text { in } \\
\Delta 1, \Delta 2, \Delta 3 \text { with normal T3, } \\
\text { T4 (cutoffs not mentioned) }\end{array}$ & Excluded & $\begin{array}{l}n=495 ; \text { age: } 23.8 \pm 3.7 \\
\text { years; GA: } \Delta 1, \Delta 2, \Delta 3\end{array}$ & $\begin{array}{l}\text { TH: } 14 \\
\text { OH: } \\
\text { SCH: } 14\end{array}$ \\
\hline 60 & $\begin{array}{l}\text { Marwaha et al. 2008; } \\
\text { Delhi, Delhi [85] }\end{array}$ & Hospital-based & $\begin{array}{c}\text { SCH: TSH }>4.2 \mathrm{mIU} / \mathrm{L} \text { in } \\
\Delta 1, \Delta 2, \Delta 3 \text { with normal T3 } \\
(3.7-7.2 \mathrm{pg} / \mathrm{ml}), \mathrm{T} 4 \\
(12.0-23.0 \mathrm{pg} / \mathrm{ml}) ; \mathrm{OH}: \\
\text { TSH }>4.2 \mathrm{mIU} / \mathrm{L} \text { in } \Delta 1, \Delta 2, \\
\Delta 3 \text { with normal T3 } \\
(<3.7 \mathrm{pg} / \mathrm{ml}), \mathrm{T} 4 \\
(<12.0 \mathrm{pg} / \mathrm{ml})\end{array}$ & Excluded & $\begin{array}{c}n=541 \text {; age: not } \\
\text { mentioned; GA: } \Delta 1, \\
\Delta 2, \Delta 3\end{array}$ & $\begin{array}{l}\text { TH: } 85 \\
\text { OH: } 7 \\
\text { SCH: } 78\end{array}$ \\
\hline 61 & $\begin{array}{c}\text { Kharkongor and Gupta } \\
\text { 1998; Meghalaya [86] }\end{array}$ & $\begin{array}{l}\text { Community- } \\
\text { based }\end{array}$ & $\begin{array}{c}\text { Hypothyroidism: } \\
\mathrm{TSH}>7 \mathrm{mIU} / \mathrm{L} \text { in } \Delta 1, \Delta 2, \\
\Delta 3\end{array}$ & Included & $\begin{array}{c}n=70 ; \text { age: } 15-25 \text { years; } \\
\text { GA: } \Delta 1, \Delta 2, \Delta 3\end{array}$ & $\begin{array}{l}\text { TH: } 1 \\
\text { OH: } \\
\text { SCH: }\end{array}$ \\
\hline
\end{tabular}

ATA: American Thoracic Association; TH: total hypothyroidism; OH: overt hypothyroidism; SCH: subclinical hypothyroidism. 


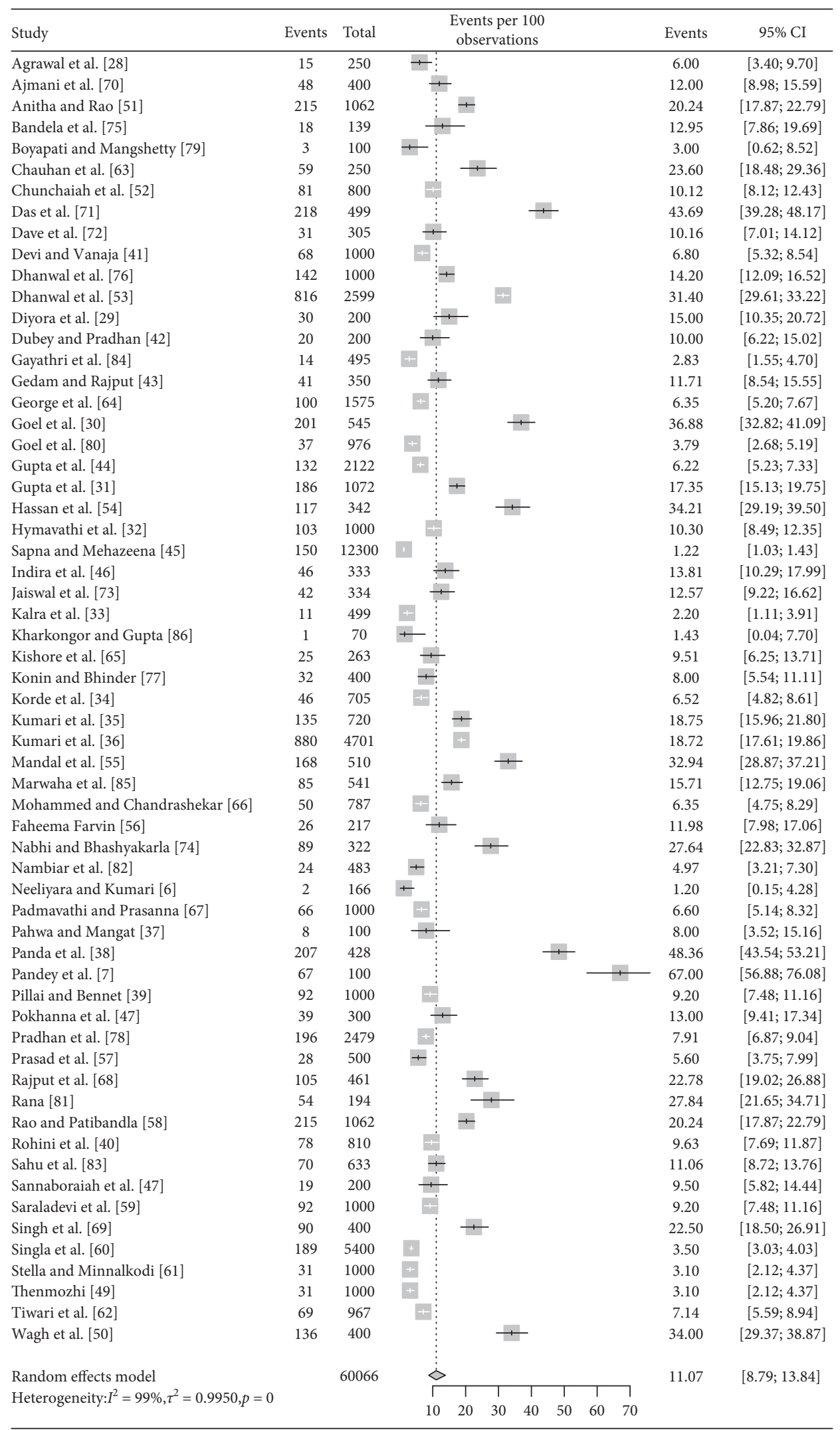

FIGURE 2: Forest plot showing pooled estimate for prevalence of overall hypothyroidism. 


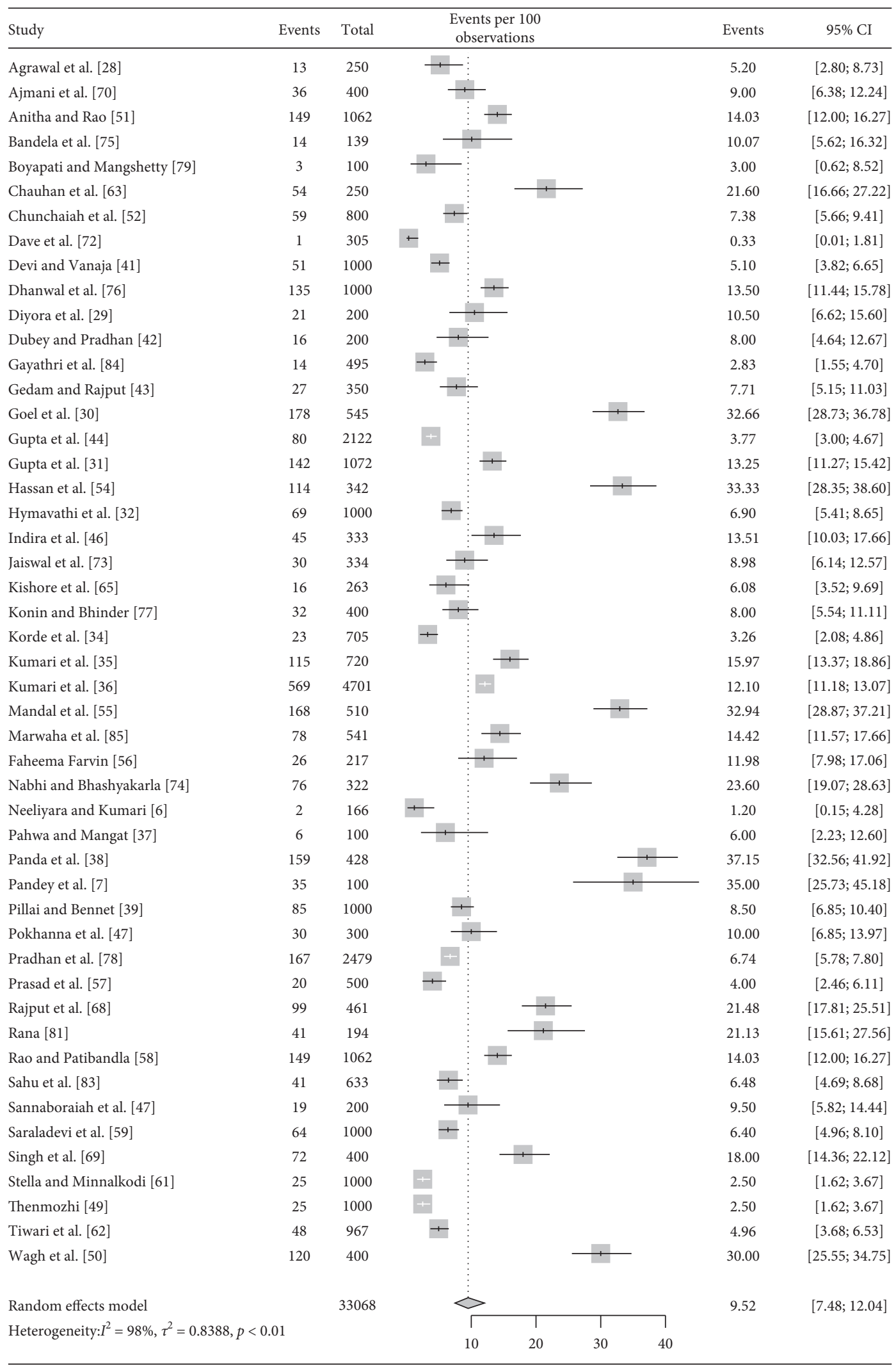

FIGURE 3: Forest plot showing pooled estimate for prevalence of subclinical hypothyroidism. 


\begin{tabular}{|c|c|c|c|c|c|c|}
\hline Study & Events & Total & $\begin{array}{c}\text { Events per } 100 \\
\text { observations }\end{array}$ & & Events & $95 \% \mathrm{CI}$ \\
\hline Agrawal et al. [28] & 2 & 250 & + & & 0.80 & {$[0.10 ; 2.86]$} \\
\hline Ajmani et al. [70] & 12 & 400 & $\vdots$ & & 3.00 & {$[1.56 ; 5.18]$} \\
\hline Anitha and Rao [51] & 66 & 1062 & $\vdots+$ & & 6.21 & {$[4.84 ; 7.84]$} \\
\hline Bandela et al. [75] & 4 & 139 & $\div$ & & 2.88 & {$[0.79 ; 7.20]$} \\
\hline Chauhan et al. [63] & 5 & 250 & + & & 2.00 & {$[0.65 ; 4.61]$} \\
\hline Chunchaiah et al. [52] & 22 & 800 & + & & 2.75 & {$[1.73 ; 4.13]$} \\
\hline Dave et al. [72] & 30 & 305 & $\longrightarrow$ & & 9.84 & {$[6.74 ; 13.74]$} \\
\hline Devi and Vanaja [41] & 17 & 1000 & + & & 1.70 & {$[0.99 ; 2.71]$} \\
\hline Dhanwal et al. [76] & 7 & 1000 & $+\vdots$ & & 0.70 & {$[0.28 ; 1.44]$} \\
\hline Diyora et al. [29] & 9 & 200 & $\vdots$ & & 4.50 & {$[2.08 ; 8.37]$} \\
\hline Dubey and Pradhan [42] & 4 & 200 & $\div$ & & 2.00 & {$[0.55 ; 5.04]$} \\
\hline Gedam and Rajput [43] & 14 & 350 & $\div$ & & 4.00 & {$[2.20 ; 6.62]$} \\
\hline Goel et al. [30] & 23 & 545 & $=$ & & 4.22 & {$[2.69 ; 6.27]$} \\
\hline Gupta et al. [44] & 52 & 2122 & + & & 2.45 & {$[1.84 ; 3.20]$} \\
\hline Gupta et al. [31] & 44 & 1072 & + & & 4.10 & {$[3.00 ; 5.47]$} \\
\hline Hassan et al. [54] & 3 & 342 & $+\vdots$ & & 0.88 & {$[0.18 ; 2.54]$} \\
\hline Hymavathi et al. [32] & 34 & 1000 & + & & 3.40 & {$[2.37 ; 4.72]$} \\
\hline Indira et al. [46] & 1 & 333 & $\leftarrow \vdots$ & & 0.30 & {$[0.01 ; 1.66]$} \\
\hline Jaiswal et al. [73] & 12 & 334 & $\div$ & & 3.59 & {$[1.87 ; 6.19]$} \\
\hline Kishore et al. [65] & 9 & 263 & $\div$ & & 3.42 & {$[1.58 ; 6.40]$} \\
\hline Korde et al. [34] & 23 & 705 & $\div$ & & 3.26 & {$[2.08 ; 4.86]$} \\
\hline Kumari et al. [35] & 20 & 720 & + & & 2.78 & {$[1.70 ; 4.26]$} \\
\hline Kumari et al. [36] & 311 & 4701 & $\vdots-$ & & 6.62 & {$[5.92 ; 7.36]$} \\
\hline Marwaha et al. [85] & 7 & 541 & + & & 1.29 & {$[0.52 ; 2.65]$} \\
\hline Nabhi and Bhashyakarla [74] & 13 & 322 & 7 & & 4.04 & {$[2.17 ; 6.80]$} \\
\hline Pahwa and Mangat [37] & 2 & 100 & $\div$ & & 2.00 & {$[0.24 ; 7.04]$} \\
\hline Panda et al. [38] & 48 & 428 & $\longrightarrow$ & & 11.21 & {$[8.39 ; 14.59]$} \\
\hline Pandey et al. [7] & 32 & 100 & & + & 32.00 & {$[23.02 ; 42.08]$} \\
\hline Pillai and Bennet [39] & 7 & 1000 & $+\vdots$ & & 0.70 & {$[0.28 ; 1.44]$} \\
\hline Pokhanna et al. [47] & 9 & 300 & $\vdots$ & & 3.00 & {$[1.38 ; 5.62]$} \\
\hline Pradhan et al. [78] & 29 & 2479 & + & & 1.17 & {$[0.78 ; 1.68]$} \\
\hline Prasad et al. [57] & 8 & 500 & + & & 1.60 & {$[0.69 ; 3.13]$} \\
\hline Rajput et al. [68] & 6 & 461 & \pm & & 1.30 & {$[0.48 ; 2.81]$} \\
\hline Rana [81] & 13 & 194 & $\vdots$ & & 6.70 & {$[3.62 ; 11.19]$} \\
\hline Rao and Patibandla [58] & 66 & 1062 & + & & 6.21 & {$[4.84 ; 7.84]$} \\
\hline Sahu et al. [83] & 29 & 633 & 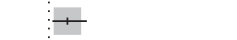 & & 4.58 & {$[3.09 ; 6.51]$} \\
\hline Saraladevi et al. [59] & 28 & 1000 & $\doteqdot$ & & 2.80 & {$[1.87 ; 4.02]$} \\
\hline Singh et al. [69] & 18 & 400 & ! & & 4.50 & {$[2.69 ; 7.02]$} \\
\hline Stella and Minnalkodi [61] & 6 & 1000 & $+\vdots$ & & 0.60 & {$[0.22 ; 1.30]$} \\
\hline Thenmozhi [49] & 6 & 1000 & $+\vdots$ & & 0.60 & {$[0.22 ; 1.30]$} \\
\hline Tiwari et al. [62] & 21 & 967 & + & & 2.17 & {$[1.35 ; 3.30]$} \\
\hline Wagh et al. [50] & 16 & 400 & $\div$ & & 4.00 & {$[2.30 ; 6.41]$} \\
\hline \multirow{2}{*}{\multicolumn{2}{|c|}{$\begin{array}{l}\text { Random effects model } \\
\text { Heterogeneity: } I^{2}=94 \%, \tau^{2}=0.7376, p<0.01\end{array}$}} & 30980 & $\dot{\diamond}$ & & \multirow[t]{2}{*}{2.74} & \multirow[t]{2}{*}[2.09;3.58]{} \\
\hline & & & $\begin{array}{r}1 \\
20\end{array}$ & $\begin{array}{c}1 \\
30 \\
30\end{array}$ & & \\
\hline
\end{tabular}

FIGURE 4: Forest plot showing pooled estimate for prevalence of overt hypothyroidism.

3.4. Publication Bias. Regarding the publication bias of pooled estimate of overall hypothyroidism, funnel plot is reasonably symmetrical on visual inspection (Figure 6). Rank correlation test results $(z=-1.73, p$ value $=0.082)$ also confirm absence of publication bias.

\section{Discussion}

The sixty-one studies included in the current review have reported prevalence of maternal hypothyroidism ranging from $1.2 \%$ to $67.0 \%[6,7]$.
This meta-analysis estimates the prevalence of hypothyroidism in pregnant women in India to be $11.07 \%$ (95\% CI: 8.79-13.84) from sixty-one studies across 60,066 study subjects. Pooled prevalence estimates of subclinical and overt hypothyroidism were 9.52\% (95\% CI: 7.48-12.04) and $2.74 \%$ (95\% CI: 2.09-3.58), respectively.

Sepasi et al. conducted a meta-analysis to report prevalence of hypothyroidism clinical and subclinical hypothyroidism in Iranian pregnant women. Prevalence estimate for hypothyroidism, clinical hypothyroidism, and subclinical hypothyroidism was $13.01 \%$ (95\% CI: 9.15-18.17), 1.35\% 
TABLE 2: Subgroup analysis of overall hypothyroidism estimates.

\begin{tabular}{|c|c|c|c|c|c|c|}
\hline Subgroup categories & Number of studies & ES & $95 \% \mathrm{CI}$ & $I^{2}(\%)$ & Between group $Q$ & Between group $p$ value \\
\hline Gestational period (trimester) & & & & & 3.47 & 0.1544 \\
\hline First & 20 & 10.99 & $8.11-14.74$ & 96.6 & & \\
\hline Second & 4 & 25.51 & $10.41-50.24$ & 98.1 & & \\
\hline Any trimester & 37 & 10.05 & $7.33-13.65$ & 99.1 & & \\
\hline Prepregnancy thyroid patients' exclusion & & & & & 0.03 & 0.8637 \\
\hline Excluded & 25 & 11.31 & $8.57-14.79$ & 97.6 & & \\
\hline Included & 36 & 10.89 & $7.73-15.14$ & 99.1 & & \\
\hline Site & & & & & 17.17 & $<0.001$ \\
\hline Hospital-based & 54 & 12.32 & $9.97-15.13$ & 98.4 & & \\
\hline Secondary data & 5 & 6.24 & $1.83-19.17$ & 99.6 & & \\
\hline Community-based & 2 & 1.27 & $0.41-3.86$ & 0.0 & & \\
\hline Coastal state & & & & & 3.55 & 0.0597 \\
\hline Yes & 31 & 8.82 & $6.45-11.94$ & 98.3 & & \\
\hline No & 29 & 13.49 & $9.77-18.34$ & 98.7 & & \\
\hline Criteria/definition & & & & & 5.04 & 0.0247 \\
\hline ATA 2011 & 35 & 13.71 & $10.52-17.67$ & 98.5 & & \\
\hline Other & 26 & 8.10 & $5.49-11.80$ & 98.8 & & \\
\hline
\end{tabular}

TAвLE 3: Subgroup analysis showing state-wise prevalence (Cochrane $Q: 747.87, p$ value: $<0.001$ ).

\begin{tabular}{lccc}
\hline States of India & Number of studies & ES (percent prevalence) & 95\% CI \\
\hline Andhra Pradesh & 6 & 10.78 & $7.80-14.72$ \\
Assam & 1 & 43.69 & $39.39-48.08$ \\
Chandigarh & 2 & 13.18 & $2.27-49.79$ \\
Chhattisgarh & 1 & 9.51 & $6.50-13.69$ \\
Delhi & 5 & 10.59 & $7.62-14.53$ \\
Gujarat & 2 & 20.82 & $13.24-31.19$ \\
Haryana & 2 & 7.60 & $1.33-33.38$ \\
Karnataka & 7 & 8.70 & $7.15-10.53$ \\
Kerala & 4 & 5.46 & $3.15-9.29$ \\
Madhya Pradesh & 3 & 14.82 & $9.66-22.06$ \\
Maharashtra & 6 & 7.55 & $3.21-16.74$ \\
Manipur & 1 & 22.5 & $18.67-26.85$ \\
Meghalaya & 1 & 1.43 & $0.20-9.45$ \\
Multiple states & 2 & 19.38 & $8.85-37.32$ \\
Odisha & 1 & 48.36 & $43.66-53.1$ \\
Punjab & 3 & 5.07 & $3.42-7.47$ \\
Sikkim & 1 & 10 & $6.54-14.99$ \\
Jammu and Kashmir & 1 & 34.21 & $29.37-39.4$ \\
Tamil Nadu & 4 & 4.23 & $2.3-7.64$ \\
Telangana & 3 & 22.01 & $18.82-25.56$ \\
Uttar Pradesh & 3 & 13.79 & 67 \\
Uttarakhand & 1 & 32.94 & $57.23-75.49$ \\
West Bengal & 1 & 20.97 \\
\hline
\end{tabular}

(95\% CI: $0.97-1.86)$, and $11.90 \%$ (95\% CI: 7.40-18.57), respectively, which are very similar to our study [87]. However, prevalence reported in our study is remarkably higher as compared to the reported prevalence of antenatal hypothyroidism in other countries [3-5]. The higher burden in the Indian context may be attributed to the iodine deficiency, prevalent in many regions of the country [88-90].

In this review, we found prevalence of hypothyroidism was lower among coastal areas as compared to studies conducted in noncoastal areas, though difference was nonsignificant $(p=0.059)$. Unnikrishnan et al. conducted a survey of hypothyroidism among adult population in 8 urban cities of India [91]. They reported significant lower prevalence of hypothyroidism in coastal cities as compared to noncoastal cities. This may be due to iodine deficiency, which is still more prevalent in noncoastal areas of India [88-90].

In our study, we documented higher prevalence of hypothyroidism in studies that used ATA 2011 criteria as compared to studies that used other criteria, and this difference is statistically significant $(p=0.024)$. This may be due to higher cutoffs of TSH for diagnosis, used by other stidies.

Primary maternal hypothyroidism is characterized by an increase in the serum TSH levels during pregnancy. It is 


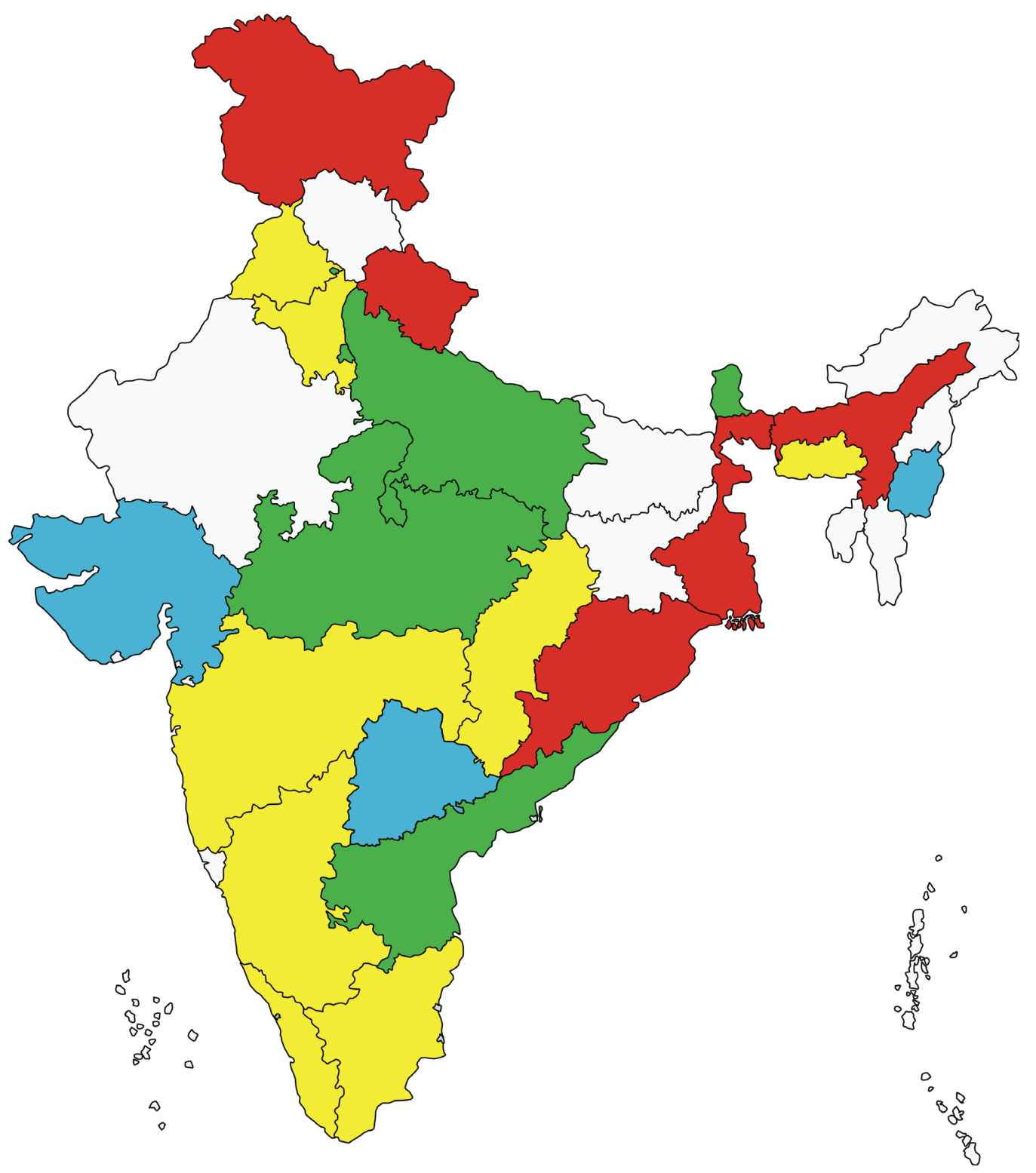

Prevalence of hypothyroidism among pregnant women

$\square 0-9 \%$

$\square 10-19 \%$

$>30 \%$

$\square 20-29 \%$

$\square$ No data available

Figure 5: Distribution of hypothyroidism across various states of India.

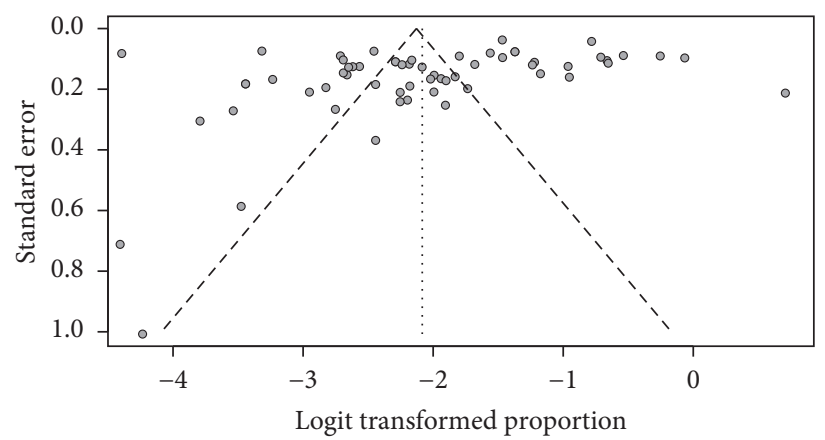

Figure 6: Funnel plot depicting publication bias of included studies. 
further classified as subclinical hypothyroidism (SCH) which has normal free T4 levels and overt hypothyroidism $(\mathrm{OH})$ which has decreased free T4 levels. This differentiation is crucial as it has clinical and management implications [2]. Maternal complications reported to be associated with overt hypothyroidism include pre-eclampsia, placental abruption, polyhydramnios, oligohydramnios, hyperemesis, gestational diabetes, premature rupture of membranes, and chronic hypertension [10-13, 92, 93]. For the fetus too, there is a high risk of fetal death, prematurity, low birth weight, congenital malformations, fetal distress, perinatal hypoxic encephalopathy, and deficit in the mental developmental coefficient $[11,14-19,93]$. Some epidemiological studies have also pointed towards the association of maternal hypothyroidism and adverse neurological outcomes in the progeny ranging from neurological cretinism, congenital hypothyroidism, to decreased intelligence quotient [20-22]. Jansen et al. observed 1981 motherchild pairs [94]. They found that both abnormal (low or high) maternal TSH values, early in pregnancy, were associated with a smaller offspring's total grey matter and cortical volume as assessed by MRI [94].

Data pertaining to effects of early management of SCH in pregnancy are emerging in latest research. Rao et al. in 2019 identified that levothyroxine supplementation significantly decreased pregnancy loss and prematurity in women with SCH [95]. However, Casey et al. conducted a RCT on cases of $\mathrm{SCH}$ in pregnancy to determine benefit of SCH treatment in pregnancy on cognitive health of offspring. They did not find significantly better cognitive outcomes in children up to 5 years of age [96]. Thus, there is inconclusive evidence of benefit of treating $\mathrm{SCH}$ in pregnancy.

Pregnancy is a state of increased thyroid hormone requirement. The majority (50-85\%) of previously hypothyroid women (on treatment) need to increase their dose of thyroid supplements post conception [5]. Pregnancy serves as a stress test for the thyroid gland, which leads to hypothyroidism in iodine deficient women or in those having limited thyroid reserve. Furthermore, risk factors such as geographical disparity (in terms of iodine-deficient regions especially across India), obesity, prior history of thyroid dysfunction, the genetic history of thyroid dysfunction, and history of autoimmune disorders also make pregnant women more susceptible to hypothyroidism [5].

TSH levels during pregnancy are lower as compared to the nonpregnant state. As per American Thyroid Association recommendations 2011 as well as endocrinology society guidelines for pregnant women, TSH levels should be within the limits of 0.1 to $2.5 \mathrm{mIU} / \mathrm{L}$ during the first, $0.2-3.0 \mathrm{mIU} / \mathrm{L}$ in second, and 0.3 to $3.0 \mathrm{mIU} / \mathrm{L}$ in the third trimester [1]. However, these guidelines were revised in 2017, and it was recommended that the first-trimester upper normal limit cutoff should be obtained by deducting " $0.5 \mathrm{mIU} / \mathrm{L}$ " from prepregnancy TSH value [2]. If this value is unknown, then $4.0 \mathrm{mIU} / \mathrm{L}$ should be taken as the upper limit of normal cutoffs.

Optimal TSH cutoffs have been a topic of controversy since long and may have a bearing on prevalence estimates [97]. European Thyroid Association in 2014 recommended that, due to geographic variation in normal TSH and thyroid hormone levels, reference range should be defined for each antenatal hospital after assessment of local population data [98]. ATA 2017 guidelines also supported these recommendations [2]. These guidelines might be the reason for nonstandardized cutoff values are being used by many studies.

However, in Indian setting, the National Guidelines for Screening of Hypothyroidism during Pregnancy 2014 have considered ATA 2011 guidelines for defining cutoffs [99]. A recent article suggested that $4.0 \mathrm{mIU} / \mathrm{L}$ as a cutoff as per the revised ATA recommendations may be too high for Indian settings, and intermediate cut-off of 3.00 should be considered [97].

4.1. Limitations of the Current Study. A rigorous and comprehensive search strategy was undertaken, which minimizes the possibility of changes in inferences drawn from our study, but there are limitations too. The review exhibits heterogeneity in terms of data. The prevalence varies with the geographic locations, ethnic disparity, and the nutritional intake of the study population. Another aspect that limits our research is the use of nonstandardized TSH cutoffs for diagnosis by studies, as only 35 studies used ATA 2011 TSH cutoffs [1]. Furthermore, preanalytical factors such as gestational age, presence of thyroid antibodies, iodine status, multiple pregnancies, ethnicity, and time of collection of TSH sample may also affect the results. Different immunoassays result in different TSH values, thereby questioning the reliability and repeatability of tests in various studies. Another crucial aspect is the circadian TSH rhythm varying in the second and third trimester of pregnancy. Considering this fact, the standardized collection of samples at the correct time may alter the results and their interpretation.

\section{Conclusion}

This review estimated that every 1 in 10 pregnant Indian women suffers from hypothyroidism. The present study has paved the way for the acceptance of universal screening in pregnant women, especially in the Indian context. The prevalence of hypothyroidism in pregnant women varies across states in India, but data is insufficient. Moreover, there are no agreed-upon guidelines for treating subclinical hypothyroidism in pregnant women. Therefore, further research is needed to fill these gaps regarding the diagnosis and management of hypothyroidism in pregnant women in a heterogeneous country like India.

\section{Data Availability}

The data used to support the findings of this study are included within the article as tables.

\section{Ethical Approval}

This is a systematic review and meta-analysis of already published articles. Hence, ethical approval and consent are not applicable. 


\section{Conflicts of Interest}

All authors declare no conflicts of interest.

\section{References}

[1] A. Stagnaro-Green, M. Abalovich, E. Alexander et al., "Guidelines of the American Thyroid Association for the diagnosis and management of thyroid disease during pregnancy and postpartum," Thyroid, vol. 21, no. 10, pp. 1081-1125, 2011.

[2] E. K. Alexander, E. N. Pearce, G. A. Brent et al., "2017 guidelines of the American Thyroid Association for the diagnosis and management of thyroid disease during pregnancy and the postpartum," Thyroid, vol. 27, no. 3, pp. 315-389, 2017.

[3] FIGO Working Group on Good Clinical Practice in MaternalFetal Medicine, "Good clinical practice advice: thyroid and pregnancy," International Journal of Gynaecology and $\mathrm{Ob}$ stetrics: The Official Organ of the International Federation of Gynaecology and Obstetrics, vol. 144, no. 3, pp. 347-351, 2019.

[4] P. Cignini, E. V. Cafà, C. Giorlandino, S. Capriglione, A. Spata, and N. Dugo, "Thyroid physiology and common diseases in pregnancy: review of literature," Journal of Prenatal Medicine, vol. 6, no. 4, pp. 64-71, 2012.

[5] R. Sahay and V. Nagesh, "Hypothyroidism in pregnancy," Indian Journal of Endocrinology and Metabolism, vol. 16, no. 3, pp. 364-370, 2012.

[6] A. M. Neeliyara and K. S. Kumari, Reproductive Health and Nutritional Profile of Women in the Coastal Areas of Alleppey District Kerala, Mahatma Gandhi University, Kottayam, India, 2015.

[7] S. K. Pandey, H. S. Pandey, and V. Kalra, "Study of prevalence of thyroid disorders in pregnant females of a tertiary care hospital of Garhwal region of Uttarakhand," Annals of International Medical and Dental Research, vol. 4, no. 4, pp. 25-27, 2018.

[8] R. Z. Klein, J. E. Haddow, J. D. Falx et al., "Prevalence of thyroid deficiency in pregnant women," Clinical Endocrinology, vol. 35, no. 1, pp. 41-46, 1991.

[9] N. K. Moog, S. Entringer, C. Heim, P. D. Wadhwa, N. Kathmann, and C. Buss, "Influence of maternal thyroid hormones during gestation on fetal brain development," Neuroscience, vol. 342, pp. 68-100, 2017.

[10] K. A. Toulis, A. Stagnaro-Green, and R. Negro, "Maternal subclinical hypothyroidsm and gestational diabetes mellitus: a meta-analysis," Endocrine Practice, vol. 20, no. 7, pp. 703-714, 2014.

[11] E. van den Boogaard, R. Vissenberg, J. A. Land et al., "Significance of (sub)clinical thyroid dysfunction and thyroid autoimmunity before conception and in early pregnancy: a systematic review," Human Reproduction Update, vol. 17, no. 5, pp. 605-619, 2011.

[12] M. Abalovich, S. Gutierrez, G. Alcaraz, G. Maccallini, A. Garcia, and O. Levalle, "Overt and subclinical hypothyroidism complicating pregnancy," Thyroid, vol. 12, no. 1, pp. 63-68, 2002.

[13] G. E. Krassas, K. Poppe, and D. Glinoer, "Thyroid function and human reproductive health," Endocrine Reviews, vol. 31, no. 5, pp. 702-755, 2010.

[14] D. Glinoer, M. F. Soto, P. Bourdoux et al., "Pregnancy in patients with mild thyroid abnormalities: maternal and neonatal repercussions," The Journal of Clinical Endocrinology \& Metabolism, vol. 73, no. 2, pp. 421-427, 1991.
[15] D. Glinoer, M. Riahi, J. P. Grün, and J. Kinthaert, "Risk of subclinical hypothyroidism in pregnant women with asymptomatic autoimmune thyroid disorders," The Journal of Clinical Endocrinology \& Metabolism, vol. 79, no. 1, pp. 197-204, 1994.

[16] M. Abalovich, N. Amino, L. A. Barbour et al., "Management of thyroid dysfunction during pregnancy and postpartum: an endocrine society clinical practice guideline," The Journal of Clinical Endocrinology \& Metabolism, vol. 92, no. 8, pp. S1-s7, 2007.

[17] W. C. Allan, J. E. Haddow, G. E. Palomaki et al., "Maternal thyroid deficiency and pregnancy complications: implications for population screening," Journal of Medical Screening, vol. 7, no. 3, pp. 127-130, 2000.

[18] N. Benhadi, W. M. Wiersinga, J. B. Reitsma, T. G. M. Vrijkotte, and G. J. Bonsel, "Higher maternal TSH levels in pregnancy are associated with increased risk for miscarriage, fetal or neonatal death," European Journal of Endocrinology, vol. 160, no. 6, pp. 985-991, 2009.

[19] R. Negro, A. Schwartz, R. Gismondi, A. Tinelli, T. Mangieri, and A. Stagnaro-Green, "Universal ScreeningVersusCase finding for detection and treatment of thyroid hormonal dysfunction during pregnancy," The Journal of Clinical Endocrinology \& Metabolism, vol. 95, no. 4, pp. 1699-1707, 2010.

[20] E. B. Man, W. S. Jones, R. H. Holden, and E. David Mellits, "Thyroid function in human pregnancy," American Journal of Obstetrics and Gynecology, vol. 111, no. 7, pp. 905-916, 1971.

[21] J. E. Haddow, G. E. Palomaki, W. C. Allan et al., "Maternal thyroid deficiency during pregnancy and subsequent neuropsychological development of the child," New England Journal of Medicine, vol. 341, no. 8, pp. 549-555, 1999.

[22] V. J. Pop, J. L. Kuijpens, A. L. van Baar et al., "Low maternal free thyroxine concentrations during early pregnancy are associated with impaired psychomotor development in infancy," Clinical Endocrinology, vol. 50, no. 2, pp. 149-155, 1999.

[23] A. Liberati, D. G. Altman, J. Tetzlaff et al., "The PRISMA statement for reporting systematic reviews and meta-analyses of studies that evaluate healthcare interventions: explanation and elaboration," BMJ, vol. 339, p. b2700, 2009.

[24] The Joanna Briggs Institute, Critical Appraisal Checklist for Analytical Cross Sectional Studies, The Joanna Briggs Institute, Adelaide, Australia, 2017, https://joannabriggs.org/sites/ default/files/2019-05/JBI_Critical_Appraisal-Checklist_for_ Analytical_Cross_Sectional_Studies2017_0.pdf.

[25] G. Schwarzer, "Meta: an R package for meta-analysis," $R$ News, vol. 7, no. 3, pp. 40-45, 2007.

[26] W. Viechtbauer, "Conducting meta-analyses in $\mathrm{R}$ with the metafor package," Journal of Statistical Software, vol. 36, no. 3, pp. 1-48, 2010.

[27] G. Schwarzer, H. Chemaitelly, L. J. Abu-Raddad, and G. Rücker, "Seriously misleading results using inverse of Freeman-Tukey double arcsine transformation in metaanalysis of single proportions," Research Synthesis Methods, vol. 10, no. 3, pp. 476-483, 2019.

[28] P. Agrawal, S. Mehta, M. Gupta, and P. Khare, "Prevalence of hypothyroidism in the first trimester pregnancy in primigravida in North India," Indian Journal of Obstetrics and Gynecology Research, vol. 6, no. 1, pp. 68-70, 2019.

[29] V. Diyora, T. Shah, and M. K. Mishra, "Thyroid dysfunction in women during first trimester of pregnancy: correlation with anti-thyroid peroxidase antibodies," Scholars International Journal of Biochemistry, vol. 2, no. 2, pp. 36-39, 2019. 
[30] B. Goel, A. Singh, P. Goel, J. Kaur, S. Singla, and M. Singla, "Evaluation of high risk screening protocol for detection of overt hypothyroidism in pregnancy," International Journal of Reproduction, Contraception, Obstetrics and Gynecology, vol. 7, no. 11, pp. 4581-4586, 2018.

[31] R. Gupta, S. Agarwal, K. Pandey et al., "A clinical study on thyroid dysfunctions in pregnancy and its effect on maternal and neonatal outcome," Journal of Evolution of Medical and Dental Sciences, vol. 7, no. 12, pp. 1520-1523, 2018.

[32] K. Hymavathi, M. D. Gottipati, T. Jakka, and T. C. Bhavani, "Routine screening of antenatal population for thyroid disorders-mandatory," International Journal of Reproduction, Contraception, Obstetrics and Gynecology, vol. 7, no. 7, pp. 2834-2839, 2018.

[33] B. Kalra, M. Choudhary, M. Thakral, and S. Kalra, "Prevalence of hypothyroidism in term pregnancies in North India," Indian Journal of Endocrinology and Metabolism, vol. 22, no. 1, pp. 13-15, 2018.

[34] V. R. Korde, S. P. Barse, and J. S. Barla, "Prevalence of thyroid dysfunctions in pregnant women: a prospective study in a tertiary care hospital in Maharashtra, India," International Journal of Reproduction, Contraception, Obstetrics and Gynecology, vol. 7, no. 8, pp. 3211-3215, 2018.

[35] A. Kumari, R. Srivastav, and S. Mitra, "Prevalence of thyroid dysfunction and its effects on fetomaternal outcome in pregnant women of eastern Uttar Pradesh, India," International Journal of Reproduction, Contraception, Obstetrics and Gynecology, vol. 7, no. 11, pp. 4379-4383, 2018.

[36] M. A. Kumari, K. Venkataramana, K. P. Leela, S. Aafreen, and A. Amrin, "Prevalence of thyroid dysfunction among antenatal women in a teaching hospital," Journal of Evolution of Medical and Dental Sciences, vol. 7, no. 4, pp. 441-444, 2018.

[37] S. Pahwa and S. Mangat, "Prevalence of thyroid disorders in pregnancy," International Journal of Reproduction, Contraception, Obstetrics and Gynecology, vol. 7, no. 9, pp. 34933496, 2018.

[38] J. Panda, A. Maji, J. Mishra, and M. Manjareeka, "Thyroid disorders in pregnancy: an exploratory study," Journal of Clinical and Diagnostic Research, vol. 12, no. 3, pp. 9-12, 2018.

[39] N. S. Pillai and J. Bennet, "Prevalence of hypothyroidism amongst pregnant women: a study done in rural set up," International Journal of Reproduction, Contraception, Obstetrics and Gynecology, vol. 7, no. 4, pp. 1586-1591, 2018.

[40] N. S. Rohini, S. N. Ravishankar, H. S. Madhuvan, S. Patil, and N. R. Rakshith, "A retrospective study of effect of thyroid disorders on obstetric and perinatal outcomes: a hospital based study," Indian Journal of Obstetrics and Gynecology Research, vol. 5, no. 2, pp. 247-252, 2018.

[41] P. U. Devi and G. Vanaja, "Maternal and perinatal outcome in antenatal women with hypothyroidism," International Archives of Integrated Medicine, vol. 4, no. 12, pp. 199-207, 2017.

[42] S. Dubey and A. Pradhan, "Thyroid disorder in antenatal women in sub-himalayan region: a need for universal screening," International Journal of Reproduction, Contraception, Obstetrics and Gynecology, vol. 6, no. 8, pp. 34453448, 2017.

[43] J. K. Gedam and D. A. Rajput, "Prevalence of thyroid disorders among patients attending the antenatal clinic at tertiary care centre, Parel, Mumbai, India," International Journal of Reproduction, Contraception, Obstetrics and Gynecology, vol. 6, no. 4, pp. 1235-1239, 2017.

[44] M. Gupta, P. Pandotra, M. Jindal, G. Jamwal, S. Goraya, and V. Gupta, "Comparative study of feto-maternal outcome in clinical and subclinical hypothyroidism," International
Journal of Reproduction, Contraception, Obstetrics and Gynecology, vol. 6, no. 5, pp. 1909-1914, 2017.

[45] I. S. Sapna and Z. Mehazeena, "Prevalence and outcome of hypothyroidism in women attending antenatal clinic at J. J. M. Medical College," International Journal of Reproduction, Contraception, Obstetrics and Gynecology, vol. 6, no. 8, pp. 3537-3539, 2017.

[46] G. Indira, T. Narasinga Rao, and Y. Premavardhini, "Prevalence of thyroid disorders in pregnancy at a teaching hospital," Journal of Evidence Based Medicine and Healthcare, vol. 4, no. 2, pp. 64-70, 2017.

[47] J. Pokhanna, U. Gupta, M. Alwani, and S. P. Tiwari, "Prevalence of thyroid dysfunction and impact on maternal and fetal outcome in central Indian pregnant women," International Journal of Reproduction, Contraception, Obstetrics and Gynecology, vol. 6, no. 10, pp. 4666-4670, 2017.

[48] A. Sannaboraiah, R. Upadhyaya, S. Garag, and S. Krishnappa, "Subclinical hypothyroidism in pregnancy and outcomes," International Journal of Reproduction, Contraception, Obstetrics and Gynecology, vol. 6, no. 4, pp. 1215-1221, 2017.

[49] D. Thenmozhi, "Prevalence of hypothyroidism in prgnancy and pregnancy outcome," IOSR Journal of Dental and Medical Sciences, vol. 16, no. 2, pp. 5-16, 2017.

[50] R. V. Wagh, M. R. Mundra, J. J. Upadhye, D. B. Telgote, S. N. Khillare, and P. S. Ramteke, "Thyroid screening in pregnancy," International Journal of Reproduction, Contraception, Obstetrics and Gynecology, vol. 6, no. 9, pp. 39563959, 2017.

[51] P. Anitha and S. Rao, "The prevalence of hypothyroidism in low income, urban pregnant women," IOSR Journal of Dental and Medical Sciences, vol. 15, no. 2, pp. 104-107, 2016.

[52] S. Chunchaiah, N. Prasad, B. M. Murali, B. M. Rupakala, and N. Rangaiah, "A prospective observational study of thyroid dysfunctions during pregnancy in a tertiary care hospital," International Journal of Reproduction, Contraception, Obstetrics and Gynecology, vol. 5, no. 11, pp. 3683-3689, 2016.

[53] D. Dhanwal, S. Bajaj, R. Rajput et al., "Prevalence of hypothyroidism in pregnancy: an epidemiological study from 11 cities in 9 states of India," Indian Journal of Endocrinology and Metabolism, vol. 20, no. 3, pp. 387-390, 2016.

[54] M. Hassan, S. K. Sheema, and S. M. S. Khan, "Prevalence of hypothyroidism in pregnant females of block hazratbal, Kashmir," International Journal of Contemporary Medical Research, vol. 3, no. 10, pp. 2841-2843, 2016.

[55] R. C. Mandal, D. Bhar, A. Das, S. R. Basunia, S. B. Kundu, and C. Mahapatra, "Subclinical hypothyroidism in pregnancy: an emerging problem in southern West Bengal: a cross-sectional study," Journal of Natural Science, Biology and Medicine, vol. 7, no. 1, pp. 80-84, 2016.

[56] N. Faheema Farvin, "A study on the prevalence of subclinical hypothyroidism among pregnant women," Master thesis, Tamilnadu Dr. M.G.R. Medical University, Chennai, India, 2016.

[57] D. Prasad, N. Nair, and K. Deepika., "A descriptive study of the prevalence of hypothyroidism among antenatal women and foetal outcome in treated hypothyroid women," International Journal of Reproduction, Contraception, Obstetrics and Gynecology, vol. 5, no. 6, pp. 1892-1896, 2016.

[58] S. Rao and A. Patibandla, "A study to find out the prevalence of hypothyroidism among pregnant women visiting ESI hospital Sanathnagar Hyderabad," Gynecology \& Obstetrics, vol. 6, no. 3, pp. 1-3, 2016.

[59] R. Saraladevi, T. N. Kumari, B. Shreen, and V. U. Rani, "Prevalence of thyroid disorder in pregnancy and pregnancy 
outcome," International Archives of Integrated Medicine, vol. 3, no. 3, pp. 1-11, 2016.

[60] M. Singla, S. Kumar Juneja, P. Tandon et al., "Maternal and foetal outcome in pregnancies complicated with hypothyroidism in Punjabi women," Journal of Evolution of Medical and Dental Sciences, vol. 5, no. 56, pp. 3826-3829, 2016.

[61] R. M. Stella and N. S. Minnalkodi, Prevalence of Hypothyroidism in Prgnancy and Pregnancy Outcome, The Tamilnadu Dr. M.G.R. Medical University, Chennai, India, 2016.

[62] D. Tiwari, R. Ruchika, A. Anchal, R. Bharti, and M. Sharma, "Prevalence of overt and subclinical hypothyroidism among Indian pregnant women and its effect on foetomaternal outcome," Journal of Evolution of Medical and Dental Sciences, vol. 5, no. 51, pp. 3342-3347, 2016.

[63] R. Chauhan, B. Sahu, and S. Khan, "Prevalence of hypothyroidism among pregnant women in Mahakaushal area and its impact on maternal and fetal outcome," International Journal of Medical and Applied Science, vol. 4, no. 1, pp. 140-147, 2015.

[64] M. George, S. M. George, and V. M. Jayasree Thankachi, "Hypothyroidism in pregnancy screen or not," Journal of Evolution of Medical and Dental Sciences, vol. 4, no. 29, pp. 4973-4978, 2015.

[65] R. Kishore, N. Mishra, and J. Yadav, "Hypothyroidism in pregnancy and its impact on maternal and fetal outcome," Journal of Evolution of Medical and Dental Sciences, vol. 4, no. 79, pp. 13849-13855, 2015.

[66] M. Z. Mohammed and K. Chandrashekar, "Clinical study of pregnancy with hypothyroidism and its outcome in tertiary care hospital," Journal of Evolution of Medical and Dental Sciences, vol. 4, no. 94, pp. 15927-15929, 2015.

[67] K. Padmavathi and C. Prasanna, "Incidence of hypothyroidism in antenatal women with maternal and perinatal outcome," IOSR Journal of Dental and Medical Sciences, vol. 14, no. 4, pp. 14-18, 2015.

[68] R. Rajput, V. Goel, S. Nanda, M. Rajput, and S. Seth, "Prevalence of thyroid dysfunction among women during the first trimester of pregnancy at a tertiary care hospital in Haryana," Indian Journal of Endocrinology and Metabolism, vol. 19, no. 3, pp. 416-419, 2015.

[69] K. P. Singh, H. A. Singh, H. Kamei, and L. M. Devi, "Prevalence of hypothyroidism among pregnant women in the sub mountain state of Manipur," International Journal of Scientific Study, vol. 3, no. 5, pp. 143-146, 2015.

[70] S. N. Ajmani, D. Aggarwal, P. Bhatia, M. Sharma, V. Sarabhai, and M. Paul, "Prevalence of overt and subclinical thyroid dysfunction among pregnant women and its effect on maternal and fetal outcome," The Journal of Obstetrics and Gynecology of India, vol. 64, no. 2, pp. 105-110, 2014.

[71] D. Das, S. J. S. Chisty, K. Barman, B. Talukdar, and U. Talukdar, "Prevalence of hypothyroidism among 1st trimester pregnant women in lower part of Assam: a pilot study," Journal of Obstetrics and Gynaecology Barpeta, vol. 1, no. 2, pp. 107-110, 2014.

[72] A. Dave, L. Maru, and M. Tripathi, "Importance of universal screening for thyroid disorders in first trimester of pregnancy," Indian Journal of Endocrinology and Metabolism, vol. 18, no. 5, pp. 735-738, 2014.

[73] N. Jaiswal, A. Melse-Boonstra, T. Thomas et al., "High prevalence of maternal hypothyroidism despite adequate iodine status in Indian pregnant women in the first trimester," Thyroid, vol. 24, no. 9, pp. 1419-1429, 2014.

[74] V. R. M. Nabhi and U. Bhashyakarla, "Prevalence of thyroid dysfunction among pregnant women in a rural teaching hospital in Telengana, south India," Scholars Journal of Applied Medical Sciences, vol. 2, no. 6B, pp. 2022-2025, 2014.

[75] P. V. Bandela, P. Havilah, M. Hindumathi, and K. D. Prasad, "Antenatal thyroid dysfunction in Rayalaseema region: a preliminary cross sectional study based on circulating serum thyrotropin levels," International Journal of Applied Biology and Pharmaceutical Technology, vol. 4, no. 4, pp. 74-78, 2013.

[76] D. Dhanwal, S. Prasad, A. Agarwal, V. Dixit, and A. Banerjee, "High prevalence of subclinical hypothyroidism during first trimester of pregnancy in north India," Indian Journal of Endocrinology and Metabolism, vol. 17, no. 2, pp. 281-284, 2013.

[77] S. S. Konin and G. K. Bhinder, "Detection of thyroid functions in early pregnancy as a universal screening," Journal of Evolution of Medical and Dental Sciences, vol. 2, no. 49, pp. 9457-9465, 2013.

[78] M. Pradhan, B. Anand, N. Singh, and M. Mehrotra, "Thyroid peroxidase antibody in hypothyroidism: it's effect on pregnancy," The Journal of Maternal-Fetal \& Neonatal Medicine, vol. 26, no. 6, pp. 581-583, 2013.

[79] A. K. Boyapati and B. Mangshetty, Study of Prevalence of Subclinical Hypothyroidism and Autoimmune Thyroiditis in Pregnant Women Attending BTGH, Rajiv Gandhi University of Health Sciences, Bangalore, India, 2012.

[80] P. Goel, J. Kaur, P. K. Saha, R. Tandon, and L. Devi, "Prevalence, associated risk factors and effects of hypothyroidism in pregnancy: a study from north India," Gynecologic and Obstetric Investigation, vol. 74, no. 2, pp. 89-94, 2012.

[81] R. Rana, Maternal Thyroid Dysfunction and Iodine Deficiency: Its Implications on Infant Development and Impact of Double Fortified Salt Supplementation, The Maharaja Sayajirao University of Baroda, Vadodara, India, 2012.

[82] V. Nambiar, V. S. Jagtap, V. Sarathi et al., "Prevalence and impact of thyroid disorders on maternal outcome in asianIndian pregnant women," Journal of Thyroid Research, vol. 2011, Article ID 429097, 6 pages, 2011.

[83] M. T. Sahu, V. Das, S. Mittal, A. Agarwal, and M. Sahu, "Overt and subclinical thyroid dysfunction among Indian pregnant women and its effect on maternal and fetal outcome," Archives of Gynecology and Obstetrics, vol. 281, no. 2, pp. 215-220, 2010.

[84] R. Gayathri, S. Lavanya, and K. Raghavan, "Subclinical hypothyroidism and autoimmune thyroiditis in pregnancy-a study in south Indian subjects," The Journal of the Association of Physicians of India, vol. 57, no. 10, pp. 691-693, 2009.

[85] R. Marwaha, S. Chopra, S. Gopalakrishnan et al., "Establishment of reference range for thyroid hormones in normal pregnant Indian women," BJOG: An International Journal of Obstetrics \& Gynaecology, vol. 115, no. 5, pp. 602-606, 2008.

[86] I. J. Kharkongor and B. B. P. Gupta, "Study on prevalence of hypothyroidism in women of reproductive age in Meghalaya, north-eastern India," Current Science, vol. 75, no. 12, pp. 1390-1393, 1998.

[87] F. Sepasi, T. Rashidian, M. Shokri, G. Badfar, F. Kazemi, and M. Azami, "Thyroid dysfunction in Iranian pregnant women: a systematic review and meta-analysis," BMC Pregnancy and Childbirth, vol. 20, no. 1, p. 405, 2020.

[88] C. S. Pandav, K. Yadav, R. Srivastava, R. Pandav, and M. G. Karmarkar, "Iodine deficiency disorders (IDD) control in India," The Indian Journal of Medical Research, vol. 138, no. 3, pp. 418-433, 2013.

[89] B. A. Laway and A. H. Zargar, "Iodine deficiency disorders in India," Journal of the Indian Medical Association, vol. 104, no. 10, pp. 554-556, 2006. 
[90] A. Majumder, A. Jaiswal, and S. Chatterjee, "Prevalence of iodine deficiency among pregnant and lactating women: experience in Kolkata," Indian Journal of Endocrinology and Metabolism, vol. 18, no. 4, pp. 486-490, 2014.

[91] A. Unnikrishnan, G. Bantwal, M. John, S. Kalra, R. Sahay, and N. Tewari, "Prevalence of hypothyroidism in adults: an epidemiological study in eight cities of India," Indian Journal of Endocrinology and Metabolism, vol. 17, no. 4, pp. 647-652, 2013.

[92] A. Kattah and V. D. Garovic, "Subclinical hypothyroidism and gestational hypertension: causal or coincidence?" Journal of the American Society of Hypertension, vol. 10, no. 9, pp. 688-690, 2016.

[93] S. Maraka, N. M. S. Ospina, D. T. O’Keeffe et al., "Subclinical hypothyroidism in pregnancy: a systematic review and metaanalysis," Thyroid, vol. 26, no. 4, pp. 580-590, 2016.

[94] T. A. Jansen, T. I. M. Korevaar, T. A. Mulder et al., "Maternal thyroid function during pregnancy and child brain morphology: a time window-specific analysis of a prospective cohort," The Lancet Diabetes \& Endocrinology, vol. 7, no. 8, pp. 629-637, 2019.

[95] M. Rao, Z. Zeng, F. Zhou et al., "Effect of levothyroxine supplementation on pregnancy loss and preterm birth in women with subclinical hypothyroidism and thyroid autoimmunity: a systematic review and meta-analysis," Human Reproduction Update, vol. 25, no. 3, pp. 344-361, 2019.

[96] B. M. Casey, E. A. Thom, A. M. Peaceman et al., "Treatment of subclinical hypothyroidism or hypothyroxinemia in pregnancy," New England Journal of Medicine, vol. 376, no. 9, pp. 815-825, 2017.

[97] S. Kalra, S. Agarwal, R. Aggarwal, and S. Ranabir, "Trimesterspecific thyroid-stimulating hormone: an Indian perspective," Indian Journal of Endocrinology and Metabolism, vol. 22, no. 1, pp. 1-4, 2018.

[98] J. Lazarus, R. S. Brown, C. Daumerie, A. HubalewskaDydejczyk, R. Negro, and B. Vaidya, "2014 European Thyroid Association guidelines for the management of subclinical hypothyroidism in pregnancy and in children," European Thyroid Journal, vol. 3, no. 2, pp. 76-94, 2014.

[99] Maternal Health Division, Ministry of Health \& Family Welfare, and Government of India, National Guidelines for Screening of Hypothyroidism during Pregnancy, Government of India, New Delhi, India, 2014, http://www.nrhmorissa.gov. in/writereaddata/Upload/Documents/National_Guidelines_ for_Screening_of_Hypothyroidism_during_Pregnancy.pdf. 